\title{
Experimental observations of seed growth and accompanying pedestal contamination in a self-seeded, soft $x$-ray free-electron laser
}

\author{
Gabriel Marcus, ${ }^{*}$ William M. Fawley, ${ }^{\dagger}$ Dorian Bohler, Yuantao Ding, Yiping Feng, \\ Erik Hemsing, Zhirong Huang, Jacek Krzywinski, Alberto Lutman, and Daniel Ratner \\ SLAC National Accelerator Laboratory, Menlo Park, California 94025, USA
}

(Received 3 June 2019; published 23 August 2019)

\begin{abstract}
Measurements of the soft x-ray, self-seeding spectrum at the Linac Coherent Light Source (LCLS) freeelectron laser generally display a pedestal-like distribution around the central seeded wavelength. This pedestal limits the spectral purity and can negatively affect some user applications not employing a postundulator monochromator. In this paper, we investigate the detailed experimental characteristics of both the amplified seed and its accompanying pedestal using data from a number of separate LCLS shifts over the 2015-2018 time period. We find that the amplified seed shows excellent wavelength stability and an exponential growth rate whose dependence upon energy detuning is consistent with theory. The pedestal's spectral distribution and integrated strength vary strongly shot by shot, independent of electron beam energy jitter. Its shot-averaged strength relative to that of the seed grows at least linearly with $z$ and can approach values of $15 \%$ or more. The pedestal is comprised of two separate components: (1) normal selfamplified spontaneous emission (SASE) whose total strength is nominally insensitive to energy detuning and laser heater (LH) strength; (2) sideband-like emission whose strength positively correlates with that of the amplified seed and negatively with energy detuning and LH strength. We believe this latter, non-SASE component arises from comparatively long wavelength (i.e., $\lambda \sim 0.3-3 \mu \mathrm{m}$ ) amplitude and phase modulations of the main seeded radiation line. Its shot to shot variability and LH sensitivity suggests an origin connected to growth of the longitudinal microbunching instability on the electron beam.
\end{abstract}

DOI: 10.1103/PhysRevAccelBeams.22.080702

\section{INTRODUCTION}

There is continual, serious interest in improving the longitudinal coherence and spectral purity of x-ray freeelectron lasers (FELs) at user facilities such as FLASH [1], Linac Coherent Light Source (LCLS) [2], SACLA [3], PAL-XFEL [4] and European XFEL [5] that employ high gain, self-amplified spontaneous emission (SASE). One promising method is so-called "self-seeding" [6-9] where the SASE radiation output from upstream undulator sections well before saturation is monochromatized to a narrow bandwidth (e.g., $\Delta \lambda / \lambda \leq 10^{-4}$ ) component that is then used to seed post-monochromator undulator sections. These latter sections are normally sufficiently long for the now narrow-band FEL radiation to reach full saturation and, typically, a post-saturation taper is applied to achieve higher powers. Figure 1 shows the particular realization of

\footnotetext{
*gmarcus@slac.stanford.edu

fawley@slac.stanford.edu
}

Published by the American Physical Society under the terms of the Creative Commons Attribution 4.0 International license. Further distribution of this work must maintain attribution to the author(s) and the published article's title, journal citation, and DOI. the soft $\mathrm{x}$-ray (SXR) self-seeding configuration used at the LCLS.

However, imperfections of the electron beam and/or of the monochromatized seed can noticeably reduce the quality of the seeded FEL output [10-16]. Early, multishot-averaged measurements of the output SXR radiation spectrum at the LCLS [9] often showed a pedestal-like contamination around the central seed. This contamination limits the spectral purity and may degrade or even prevent certain user applications when a post-undulator monochromator cannot be used (e.g., due to its low efficiency or to avoid radiation pulse lengthening) for cleaning the spectral content. A post-undulator monochromator is also not applicable for two-color schemes where one pulse is seeded. An example of such contamination is presented in panel (a) of Fig. 2 that plots the spectral distribution function for a typical self-seeded data set, as determined by binning the spectra of individual shots according to their individual electron beam energy $E_{B}$. Here one can observe different regions corresponding to the amplified seed, pedestal, and "normal" SASE, with the latter most obvious when $E_{B}$ is sufficiently removed from FEL resonance to eliminate seed amplification in the second undulator stage. Similar pedestal-like spectra have also been observed in the hard $\mathrm{x}$-ray self-seeding spectrum at LCLS [8]. In that situation, however, downstream users are less sensitive to such 


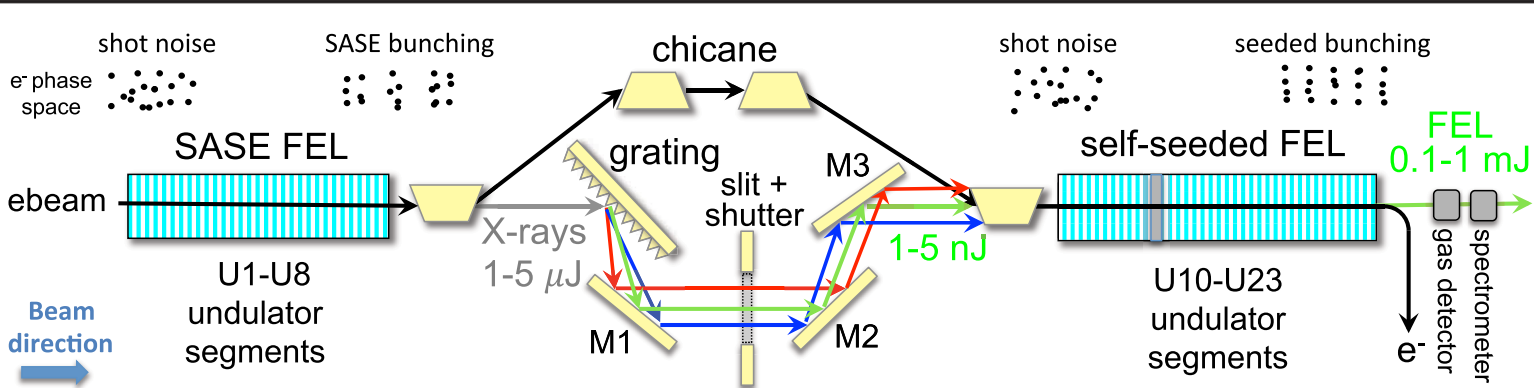

FIG. 1. Schematic of SXR self-seeding layout at LCLS (not to scale) adapted from Ref. [9]. The SXR self-seeding chicane and monochromator are at undulator segment position U9 while the hard x-ray self-seeding chicane and crystal-based monochromator (neither used in this experiment) are located at position U16.
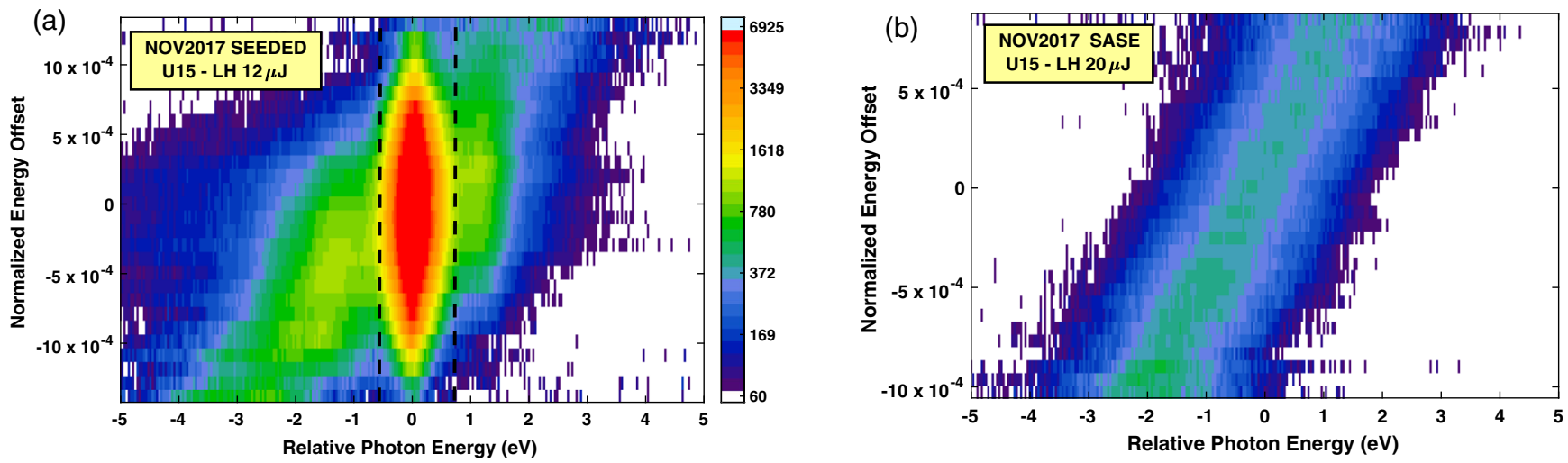

FIG. 2. Representative LCLS spectral distribution functions $S\left(\Delta E_{\text {photon }}, \Delta E_{B}\right)$ at 1-keV photon energy for both a (a) 1-keV self-seeded and a (b) SASE (blocked seed) dataset. Both datasets were obtained at effective undulator location U15 and correspond to 6 undulator segments of growth following the SXR self-seeding monochromator located at position U9. The false color scales are identical and follow a (nonlinear) $\operatorname{arcsinh}(S / 100$.) relation. In this paper, the spectral region within the dashed black lines $( \pm 0.74 \mathrm{eV})$ is defined as the amplified seed while that exterior out to $4.48 \mathrm{eV}$ corresponds to the pedestal.

contamination because a highly efficient, crystal-based monochromator in the hard x-ray user beamline filters out wider bandwidth pedestal components.

Following Ref. [9], further experimental studies ruled out the possibility that the LCLS SXR pedestals originate from spectrometer noise or the monochromator optics. Instead there are strong indications that in part the pedestal is associated with microbunching instability (MBI) modulations of the electron beam's longitudinal phase space. MBI is mostly induced by longitudinal space charge forces within the long-distance acceleration and drift sections $[17,18]$ prior to the FEL undulator. Recently, it has been directly observed at the LCLS $[19,20]$. We also note a relevant experiment carried out at the FERMI facility that intentionally seeded microbunching in the low energy, laser heater region of the linac and successfully generated multicolor, extreme-ultraviolet FEL pulses [21].

In this paper we present extensive new experimental results from the LCLS based on data from 4 separate shifts that studied the nature and quantitative behavior of pedestal formation in the self-seeded, SXR spectrum at 1.0-keV and $750-\mathrm{eV}$ central photon energies. Table I summarizes the typical electron beam and FEL operating parameters; the values to the left in the middle column refer to the $1-\mathrm{keV}$ shifts of June 2015, October 2016, and November 2017, while those to the right in brackets refer to the last shift of October 2018 at $750 \mathrm{eV}$. The last two shifts are particularly noteworthy in that the LCLS laser heater (LH) was both carefully aligned and varied in strength to examine its effects on the pedestal emission. These two shifts also employed a "blocked seed" method that allowed us to measure the growth of SASE downstream of the selfseeding monochromator separately from that of the amplified seed; an example is shown in Fig. 2(b). This permitted us to get an independent, quantitative estimate of the strength of the SASE component of the pedestal. Appendix A gives much greater detail concerning the experimental procedures, relevant downstream spectrometer and camera characteristics, and finally crucial differences between the four individual shifts.

The remainder of the paper is organized as follows. In Sec. II we examine the behavior of the amplified seed line, including its gain and spectral stability characteristics. Delineating these characteristics are useful in our opinion 
to understand the base seed structure which is then modified by the pedestal emission. We then turn in Sec. III to the behavior of the pedestal including its stochastic nature, its relative integrated strength as a function of undulator length, the relative strengths of its SASE and non-SASE components, and sensitivity to the LCLS LH strength. Section IV investigates certain spectral correlation properties of the pedestal, drawing a contrast to what is expected from pure SASE. In Sec. V we conclude with a short discussion of these results and their consequences for improving self-seeding operations at high rep-rate facilities such as the European XFEL and LCLS-II. A separate paper [22] currently under preparation will discuss the longitudinal mode statistical properties of both the seed and pedestal radiation.

\section{CHARACTERISTICS OF THE AMPLIFIED SELF-SEEDED LINE}

\section{A. Amplified seed energy exponential growth}

For the three shifts operated at $1.0-\mathrm{keV}$ photon energy, we explored the self-seeding performance as a function of the number of effective undulator sections acting beyond the self-seeding monochromator. As displayed in Fig. 3, the strength of the main self-seeding line initially grows exponentially with undulator number in the U11-U15 region and then shows saturation effects by approximately undulator section U18. In order to minimize energydetuning effects associated with the shot-to-shot energy jitter of the room-temperature SLAC linac, we restricted the data used in Fig. 3 to those shots whose normalized energy offset was $\leq 1.6 \times 10^{-4}$. Similar energy filtering is used in following plots where either photon energy or effective undulator length is the independent variable. All three shifts have similar exponential growth rates with the effective

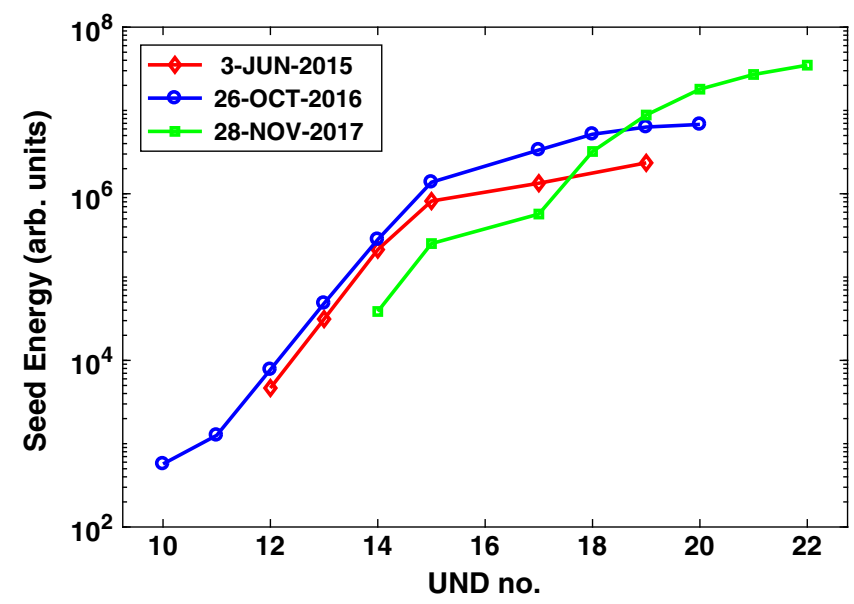

FIG. 3. Strength of the amplified self-seeded spectral line as a function of the last active undulator segment for the three 1-keV experimental shifts. The monochromatized seed is inserted at the entrance to segment U10. The signal level is derived from the post-undulator spectrometer CCD camera image.

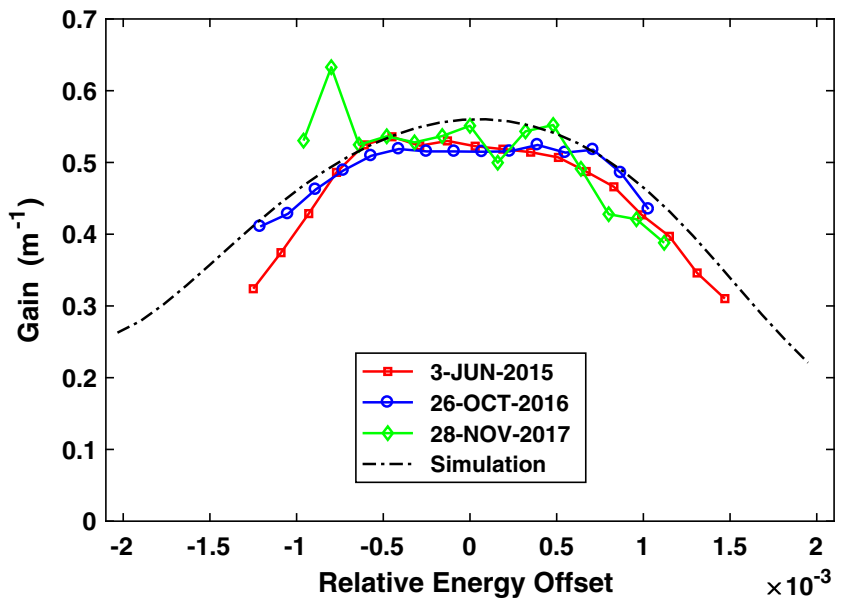

FIG. 4. Experimental and computed exponential gain coefficient for the amplified seed energy versus relative e-beam energy. Statistical fit errors (RMS) are generally $\leq 0.02 \mathrm{~m}^{-1}$ for the JUN2015 and OCT2016 data. The black line labeled "Simulation" refers to time-steady GINGER code results.

input power in the seed wavelength region (as determined by extrapolation backwards to U9) being approximately 4 to 5 orders of magnitude below the apparent FEL saturation power. Typical normalized RMS errors range from $\approx 0.4$ at the earliest undulators decreasing to $\approx 0.15$ or smaller at the most distant undulators where saturation effects become important.

The shot-to-shot jitter in the output electron beam energy $E_{B}$ from the LCLS linac has one useful benefit in that it allows examination of both the effective FEL detuning gain curve and, as will be discussed later in Sec. III, the relative strength and spectral properties of the pedestal as a function of $E_{B}$. Figure 4 plots the computed power gain coefficient of the amplified seed for each of the $1-\mathrm{keV}$ shifts determined via linear regression as a function of $E_{B}$. To minimize FEL radiation and CCD detector saturation effects, the analysis employed data from segments U12 through U15 for the June 2015 and October 2016 shifts and U14-U15 for the November 2017 shift. Each undulator segment was presumed to have an active length of $3.36 \mathrm{~m}$. The figure also includes the predicted gain curves according to time-steady numerical simulations with the GINGER code [23] using the electron beam parameters of Table I. The empirical gain curves of all three experimental shifts agree well with simulation predictions, although near peak gain they are somewhat flatter with respect to energy offset. This flattening could perhaps be caused by an effective linear energy chirp in the actual electron beam and/or a mild CCD saturation effect.

\section{B. Development of the shot-averaged spectrum vs effective undulator length}

Besides the exponential growth rate, one may also study the development of the shot-averaged spectrum with 
TABLE I. Expt. Parameters.

\begin{tabular}{lcl}
\hline \hline Parameter & Value & Unit \\
\hline Electron beam energy $E_{B}$ & $4.74[4.10]$ & $\mathrm{GeV}$ \\
Electron beam current $I_{B}$ & $1.3[1.4]$ & $\mathrm{kA}$ \\
Bunch charge $Q$ & $135[145]$ & $\mathrm{pC}$ \\
Slice energy spread $\sigma_{E}$ & 1.5 & $\mathrm{MeV}$ \\
Normalized emittance $\varepsilon_{N}$ & 0.6 & $\mu \mathrm{m}$ \\
Average focusing $\beta$ & 11 & $\mathrm{~m}$ \\
Seeding photon energy $\hbar \omega_{0}$ & $1.0[0.75]$ & $\mathrm{keV}$ \\
Expt. gain length $L_{G}$ & 1.9 & $\mathrm{~m}$ \\
Derived 1D FEL parameter $\rho$ & $1.1 \times 10^{-3}$ & \\
\hline \hline
\end{tabular}

undulator length. In Fig. 5 we plot the fractional enclosed signal as a function of integrated bandwidth for various effective undulator lengths in the October 2016 shift; for this figure only, the data was corrected for point spread function (PSF) blurring (see Appendix B for a discussion of our PSF determination). Beyond location U14 the spectral bandwidth begins to grow significantly with increasing undulator length, as shown by its effective FWHM measure (i.e., defined as the minimum bandwidth that contains $76.1 \%$ of the integrated signal) increasing from $250 \mathrm{meV}$ at $\mathrm{U} 14$ to more than $500 \mathrm{meV}$ at U17. As will be discussed in Sec. III, we believe this increase is predominantly driven by pedestal growth. At U13 and U14, the inner portions of the spectrum are reasonably well fit by a Gaussian curve with a $220 \mathrm{meV}$ FWHM. With no correction for PSF blurring, the value for the best fit Gaussian FWHM increases to $350 \mathrm{meV}$.

\section{Amplified seed wavelength stability}

The self-seeding scheme is presumed to have the intrinsic virtue of an essentially fixed output wavelength set by central line wavelength $\lambda_{0}$ of the monochromator-chicane system.

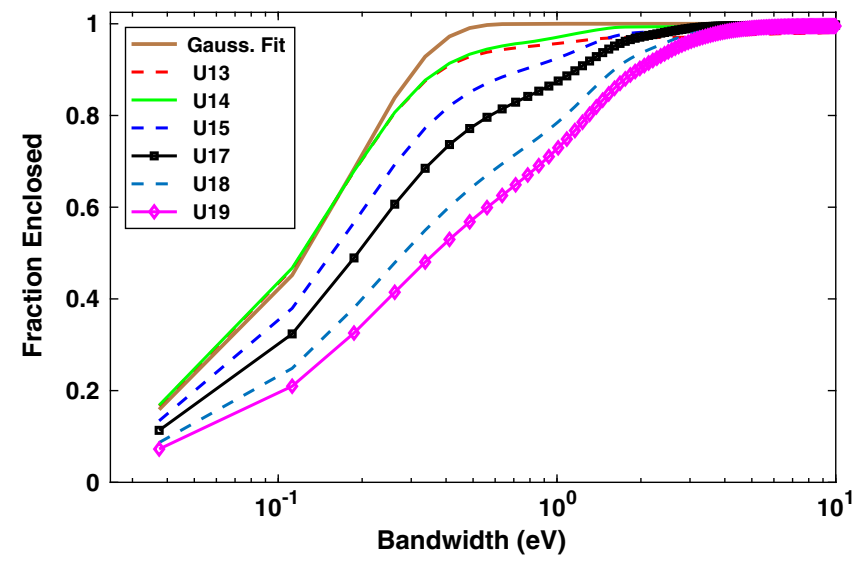

FIG. 5. Fraction of spectral energy as a function of integrated bandwidth for different effective undulator lengths for the October 2016 shift. For this data PSF-blurring has been removed via deconvolution. The curve labeled "Gauss. Fit" corresponds to a Gaussian with a FWHM of $220 \mathrm{meV}$.

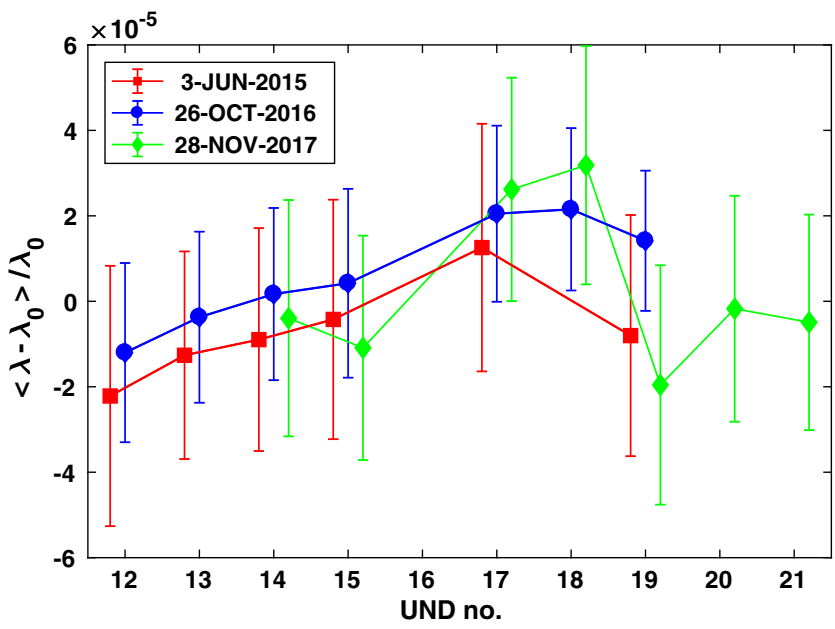

FIG. 6. Relative mean wavelength normalized to the nominal central value as a function of effective undulator \# for the $1.0 \mathrm{keV}$ experimental shifts. The error bars correspond to the RMS deviations of the individual shot values from the mean $\bar{\lambda}$ at each undulator. To improve error bar legibility, the curves have been slightly offset both horizontally and vertically from each other.

This is in contrast to SASE output whose center-of-mass wavelength $\bar{\lambda}$ can vary stochastically from shot to shot. This presumption is verified in general as shown in Fig. 6 where we plot the shot-averaged $\langle\bar{\lambda}\rangle$ relative to a fixed value for each of the three $1.0 \mathrm{keV}$ experimental shifts. We again selected shots lying in the same central electron beam energy regions previously used in Fig. 3. For each individual shot, we defined $\bar{\lambda}$ as the center-of-mass value determined over a window whose $\lambda$ width was approximately equal to the shot-averaged FWHM bandwidth. Use of much larger averaging windows can make $\bar{\lambda}$ sensitive to pedestal signals (see Sec. III), especially at effective undulator lengths beyond U15. From Fig. 6 one sees that $\langle\bar{\lambda}\rangle$ for each shift remains more or less constant with undulator length to within $\pm 2 \times 10^{-5} \lambda_{0}$. This value is nearly $50 \times$ smaller than the derived FEL parameter $\rho$, a typical measure of SASE normalized spectral width just before saturation. Moreover, the RMS scatter of $\bar{\lambda}$ at a given undulator is generally less than $3 \times 10^{-5} \lambda_{0}$ indicating a very high degree of spectral stability from shot to shot. There was somewhat greater wavelength stability for the 1-keV October 2016 shift as its normalized RMS scatter value is approximately 30\%-50\% less than the other two datasets.

Although $\bar{\lambda}$ is more or less constant for a restricted subset of individual shots whose e-beam energy lies within a narrow region, when considering all shots at a given undulator section, some of the experimental shifts showed a small but definite shot-to-shot correlation between $\bar{\lambda}$ and $E_{B}$ (i.e., "frequency pulling"). The magnitude of the apparent slope between $\Delta\langle\bar{\lambda}\rangle$ and $\Delta\left\langle E_{B}\right\rangle$ was generally less than $2 \%$ of what would be true for SASE, again emphasizing the high stability of $\bar{\lambda}$ exhibited by SXR self-seeding at LCLS. Appendix C explores this issue in greater detail. 


\section{PEDESTAL GROWTH PROPERTIES AND SPECTRAL CONTENT}

Beginning with the earliest SXR self-seeding operations at LCLS [9], there has been evidence of a generally low relative power but broad wavelength pedestal surrounding the amplified seed spectral region. In this section we discuss various characteristics of this pedestal, mainly concentrating upon 1-keV data taken in October 2016 (for which the large shot number gives excellent statistics) and November 2017 (for which the inclusion of "blocked seed" SASE datasets and variation of the laser heater power allow one to examine more quantitatively the strength of the non-SASE pedestal component). For purposes of pedestal analysis and discussion, we define the central, amplified seed region as those wavelengths lying within $0.74 \mathrm{eV}\left(\approx \pm 3 \sigma_{\mathrm{RMS}}\right)$ of the shotand intensity-averaged $\bar{\lambda}$, and the lower and upper pedestal regions as $\pm[0.744 .48] \mathrm{eV}$ from $\bar{\lambda}$. In reality, there is additional pedestal spectral content well within this inner $0.74 \mathrm{eV}$ boundary, but without accurate correction for PSF blurring, it is difficult to distinguish with high confidence the pedestal emission from that of an amplified seed whose spectral profile is somewhat non-Gaussian.

\section{A. Shot-to-shot variation of the pedestal spectra}

In principle, a spectral pedestal might arise from a constant, shot-independent instrumental optics and/or diagnostic effect such as PSF-induced "leakage" from the main seeded line into adjacent pixel regions of the post-undulator spectrometer CCD. However, inspection of individual shots for a given effective undulator length shows that the relative pedestal power and its spectral distribution are highly variable and thus cannot arise solely from instrumental or diagnostic effects. Such variations are apparent in Fig. 7 where spectra from both seeded and SASE datasets normalized to the mean seed level are shown. Although the electron beam energies for the selected shots lie very close to that corresponding to peak seed gain, both the pedestal emission and to a lesser extent SASE are larger on the low photon energy side of the central seed. In fact this low energy preference for the pedestal emission seems to be a general property of all the different $1.0 \mathrm{keV}$ experimental shifts, especially at shorter effective undulator lengths. For the October 2016 data, at longer effective undulator lengths for which the seed reaches fairly deep saturation and where the undulator strength $K$ is tapered, there is a reversal in which the high energy side of the pedestal seems to be stronger. This reversal may be due in part to shots whose post-monochromator seed strength was statistically low and therefore for which the taper would be stronger than optimum, thus moving the center of the shot-specific FEL gain curve to somewhat higher photon energies.

\section{B. Increase of fractional pedestal power with effective undulator length}

One of the most obvious features of the pedestal contamination was that as the effective undulator length $z$ increased,

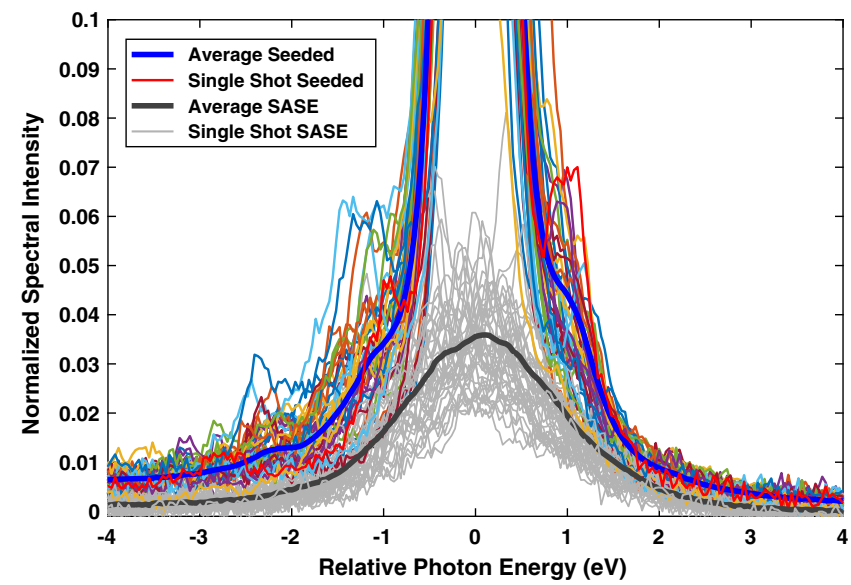

FIG. 7. Average and individual shot spectra at the end of U19 for the November 2017 shift from both self-seeded (colored lines) and blocked seed (i.e., SASE only; grey lines) datasets; the LH energy was $12 \mu \mathrm{J}$. The individual shots were randomly selected from those whose $E_{B}$ fell in the central FEL resonance region. All spectra are normalized to the peak value of the average spectrum of the $E_{B}$-filtered self-seeded dataset. There is no correction here nor in the following plots for PSF-induced "leakage" into the pedestal region from the amplified seed.

so did the pedestal strength relative to the amplified seed. As displayed in Fig. 8, linear or somewhat faster growth with $z$ was true for all the separate experimental shifts. The rate of fractional increase was somewhat slower in the November 2017 data for which the laser heater effectiveness was optimized (see the following subsection for further discussion of pedestal sensitivity to laser heater strength). Inspection of the various curves's minima suggests there is a residual pedestal fraction in the approximate range of 0.10 to 0.13 , which we attribute nearly entirely to a combination of SASE and PSF leakage. As discussed in Appendix B, measurements

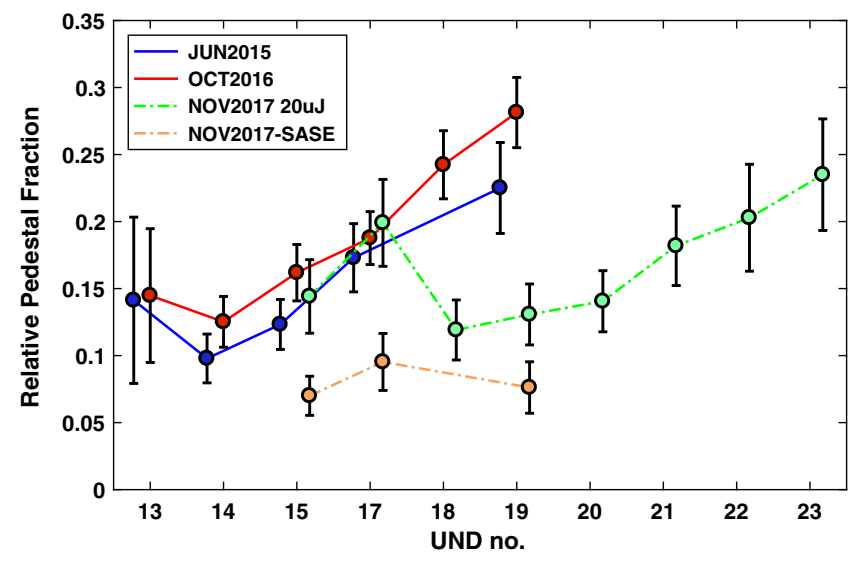

FIG. 8. Pedestal region strength normalized to that of total emission as a function of undulator position. The Nov. 2017 SASE data are normalized to the total emission of the corresponding self-seeded dataset obtained at the same LH $20 \mu \mathrm{J}$ energy. The error bars refer to RMS values. 


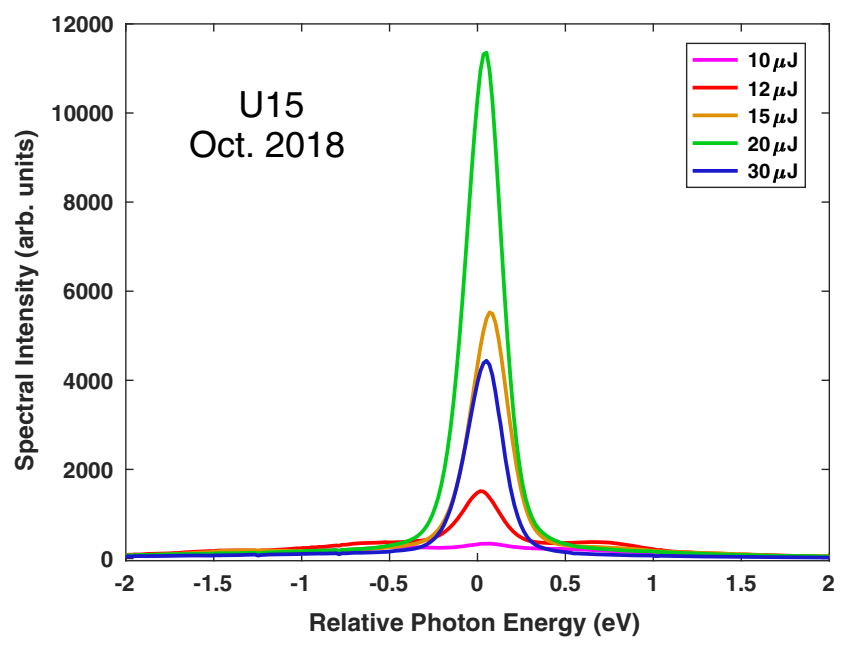

(a)

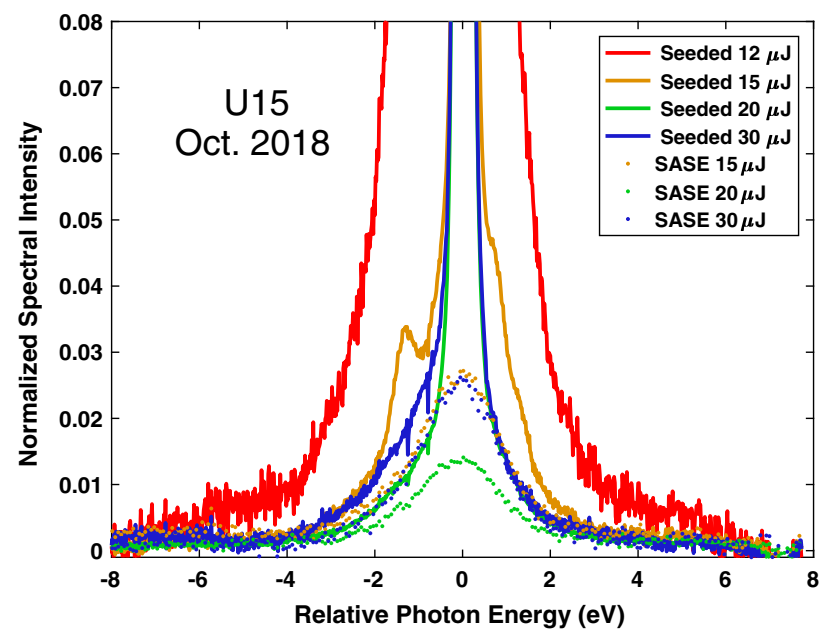

(b)

FIG. 9. (a) Oct. 2018 averaged self-seeded spectra at U15 taken at various LH energies. (b) Both self-seeded and SASE ("blocked seed") averaged spectra normalized to the peak value of the average amplified seed intensity corresponding to each LH energy value.

suggest a normalized PSF leakage value of $0.05-0.07$; blocked seed data of November 2017 (dot-dashed orange line in Fig. 8) suggests that the pure SASE contribution is $\approx 0.07$ for the LH energy of $20 \mu \mathrm{J}$. From U18 to U21 to U23, the relative pedestal fraction grows from 0.12 to 0.18 to 0.24 , illustrating that the underlying growth mechanism dominates the pedestal by U21 and beyond.

Regarding the October 2016 shift, CCD saturation effects at U17 and beyond will increase the apparent pedestal fraction, possibly by as much as a factor of 1.5 by U19 (this is not an issue for the Nov. 2017 data in which the post-undulator gas absorption cell was used to avoid such saturation effects). In the Nov. 2017 data there is an anomalous jump in both the pedestal and SASE fractional strength at U17 relative to their values at U15 and U18. Examination of Fig. 3 shows a similar drop in the amplified seed strength at $\mathrm{U} 17$ relative to that predicted by simple interpolation between $\mathrm{U} 15$ and U18. This suggests that a possible incorrect experimental setting of the phase shifter in the U16 drift section led to destructive interference at $\lambda_{o}$ in the $\mathrm{U} 17$ section, consequently raising the relative pedestal and SASE strength.

\section{Variation of the pedestal and SASE strength with laser heater power}

Both the 1.0-keV November 2017 and 750-eV October 2018 experimental shifts explored the impact of the $\mathrm{LH}$ energy upon the pedestal properties. The 2017 shift established that at all effective undulator lengths studied the fractional pedestal level did in fact strongly decrease as the LH pulse energy increased stepwise from 12 to $20 \mu \mathrm{J}$. A further increase to $30 \mu \mathrm{J}$ generally led to little additional reduction of the pedestal level. The 2018 shift confirmed these results over a wider LH energy range, albeit with such data taken only at U15.
Figure 9(a) shows the average spectra obtained in October 2018 for various LH energies. The maximum average FEL pulse energy occurs at an LH energy of $20 \mu \mathrm{J}$. Below this point the pulse energy rapidly drops and, for a LH energy of $10 \mu \mathrm{J}$, essentially disappears. We attribute this behavior at very low LH energies to large MBI growth upstream of the LCLS undulator that increases the effective incoherent energy spread $\sigma_{E}$, strongly reducing the FEL gain. When the LH pulse energy increases from 20 to $30 \mu \mathrm{J}$, the radiation pulse energy also drops, presumably because now the $\mathrm{LH}$ contribution has increased the total $\sigma_{E}$ enough to once again noticeably degrade the gain.

Figure 9(b) displays at various LH energies both the seeded and blocked seed (i.e., SASE only) spectra normalized to the peak seed intensity. The relative SASE levels for the 15 and $30 \mu \mathrm{J} \mathrm{LH}$ energies are essentially identical; the value at $20 \mu \mathrm{J}$ is $50 \%$ lower (wavelength-integrated

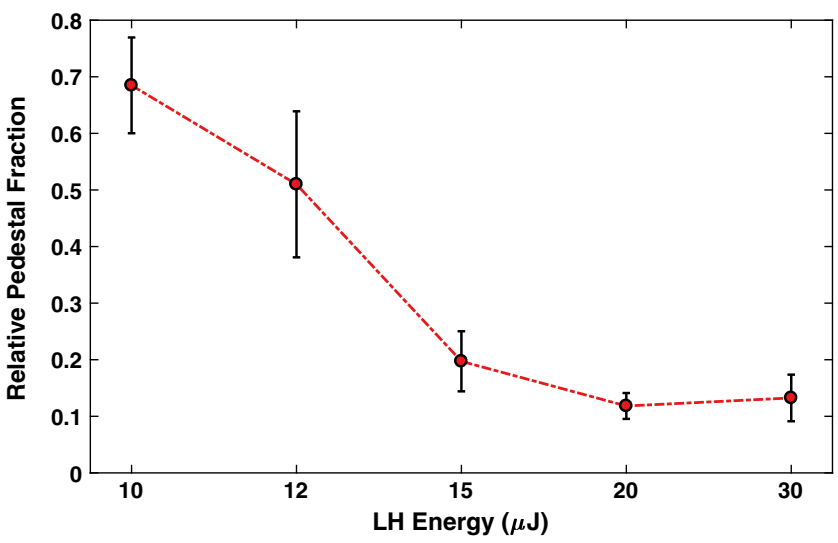

FIG. 10. Pedestal region strength normalized to total seed plus pedestal emission at U15 as a function of LH energy for the Oct. 2018 shift. The error bars refer to RMS values. 
(a)

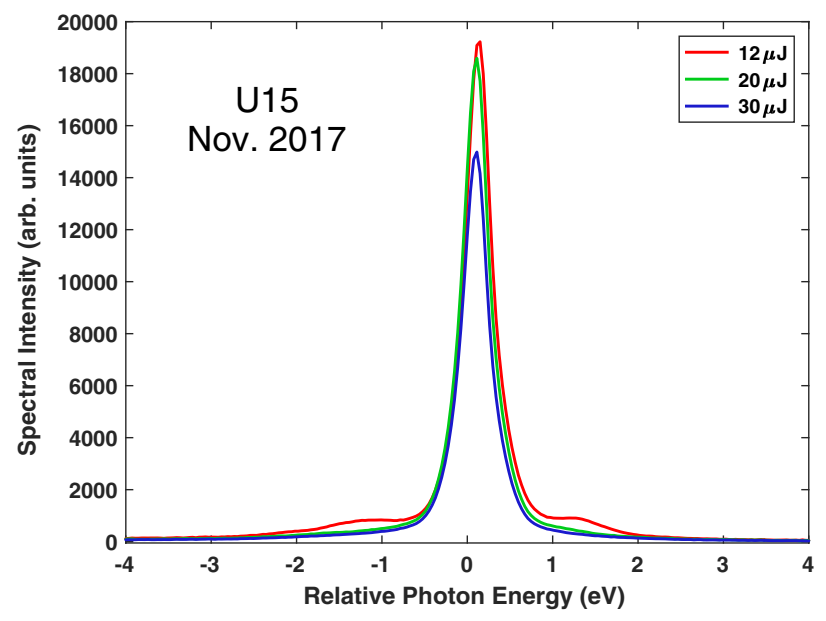

(c)

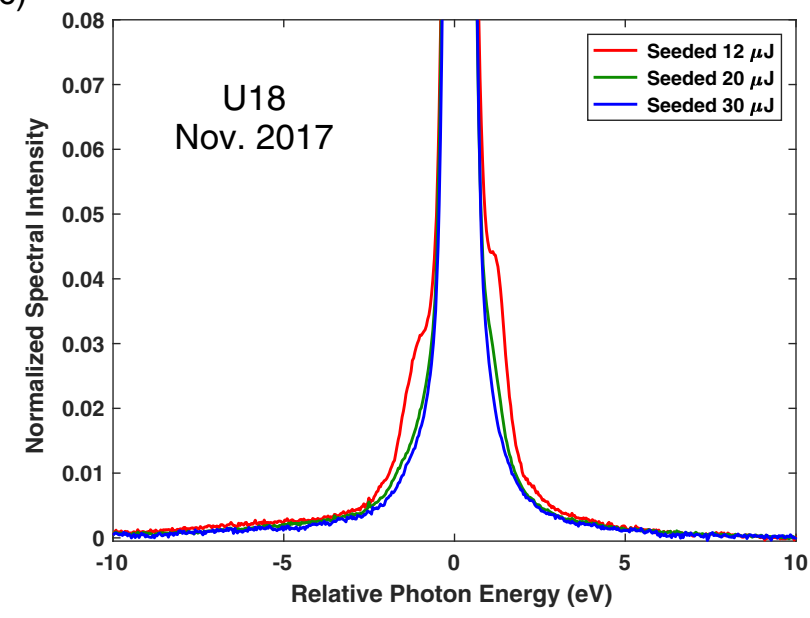

(b)

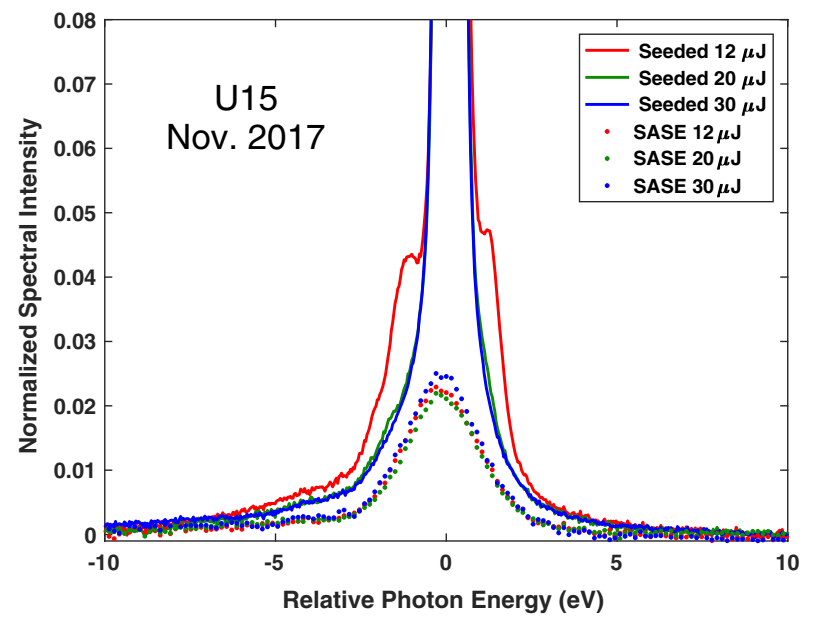

(d)

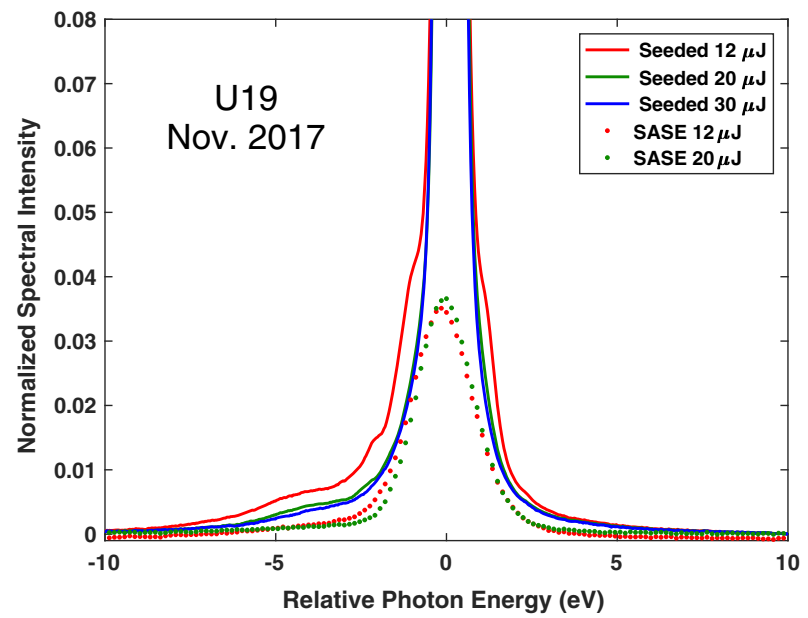

FIG. 11. Average self-seeded and SASE spectra obtained in November 2017 at various laser heater energies for 3 different undulator lengths. With the exception of (a), the spectra are normalized to the peak amplified seed intensity at each LH energy.

fractional strength 0.10 as compared with 0.16). This again suggests that the overall FEL gain was greatest at $20 \mu \mathrm{J}$ heating, thus maximizing the narrowband, monochromatized seed level at U10 relative to the wideband SASE initiated by the post-dispersion section shot noise microbunching (whose average level at entrance U10 should be more or less independent of the LH heating). The relative pedestal strength steadily decreases as the LH energy is increased from 12 to $20 \mu \mathrm{J}$, followed by a small, statistically insignificant increase at $30 \mu \mathrm{J}$. Quantitatively, this behavior is confirmed by plotting the normalized pedestal fraction versus LH energy (see Fig. 10) where the minimum value of $\approx 0.12$ occurs at $20 \mu \mathrm{J}$ heating and likely consists nearly entirely of SASE ( $\approx 0.07$ according to the blocked seed dataset) and spectrometer system PSF leakage of seed emission into the pedestal wavelength region.

These October 2018 results suggest a Goldilocks-like optimum for setting the LH energy in self-seeding mode: Too hot suppresses the MBI component of the pedestal but also both reduces the wanted narrowband, output seed intensity and decreases its contrast ratio relative to that of wideband SASE generated beyond the self-seeding monochromator at position U9. Too cool permits large MBI growth resulting in too great a total $\sigma_{E}$ at undulator entrance, possibly to the point that all FEL components (i.e., SASE and seed) can become suppressed. Just right an LH level sufficiently suppresses MBI to obtain a clean FEL output spectrum, but not so much that the pulse energy drops appreciably.

The November 2017 shift data generally confirms the effectiveness of LH damping of pedestal contamination over a wide range of effective undulator lengths. Figure 11(a) shows the average self-seeded spectra at U15 for three LH energy values. Here the FEL intensities at 12 and $20 \mu \mathrm{J}$ LH energies are nearly identical while that at $30 \mu \mathrm{J}$ is about $20 \%$ less, again suggesting that the heating has reduced the exponential gain. The normalized self-seeded and SASE spectra at U15 shown on an expanded vertical scale in Fig. 11(b) indicate that the SASE-to-seed levels in 
this shift are relatively insensitive to LH energy. However, the total pedestal contamination is much greater at $12 \mu \mathrm{J}$ than at the two higher LH energy values and is also much greater than the apparent SASE level. Even at the highest LH energy of $30 \mu \mathrm{J}$, the non-SASE component of the pedestal appears dominant at photon energies that are offset $\approx 1.8 \mathrm{eV}$ or greater from the central seed position at $1.0 \mathrm{keV}$.

The shapes of the seeded and SASE spectra for effective undulator lengths of U18 and U19 [Fig. 11(c),(d)] are qualitatively similar to those at U15 [Fig. 11(b)]. However, the relative SASE level has increased and the overall pedestal emission shifts predominantly to the red side of $\lambda_{o}$. These changes are likely due to FEL saturation effects with the FEL-related increase in $\sigma_{E}$ reducing the amplified seed growth relative to that of SASE in the blocked seed datasets whose $\sigma_{E}$ has likely grown little from U10 to U19. Similarly, the average e-beam energy at U19 in the seeded shots would be somewhat lower than that of the blocked seed shots; this should favor enhanced pedestal emission to the red for the untapered undulator configuration used in Nov. 2017.

\section{Variation of the relative pedestal power with energy detuning}

For all four experimental shifts studied here, the pedestal's integrated spectral energy and wavelength distribution showed systematic variations with electron beam energy $E_{B}$. Figure 12 shows such behavior during the November 2017 shift at two particular undulator lengths for which we also have independent, $\lambda$-resolved measures of the underlying SASE strength. In each of the plots once the normalized energy detuning becomes of order $\rho \approx 1 \times 10^{-3}$, the seed strength becomes quite small and the total pedestal strengths for both the low ("LoE Ped") and high ("HiE Ped") photon energy regions approach the corresponding SASE strengths in the lower and upper pedestal wavelength regions as shown

(a)

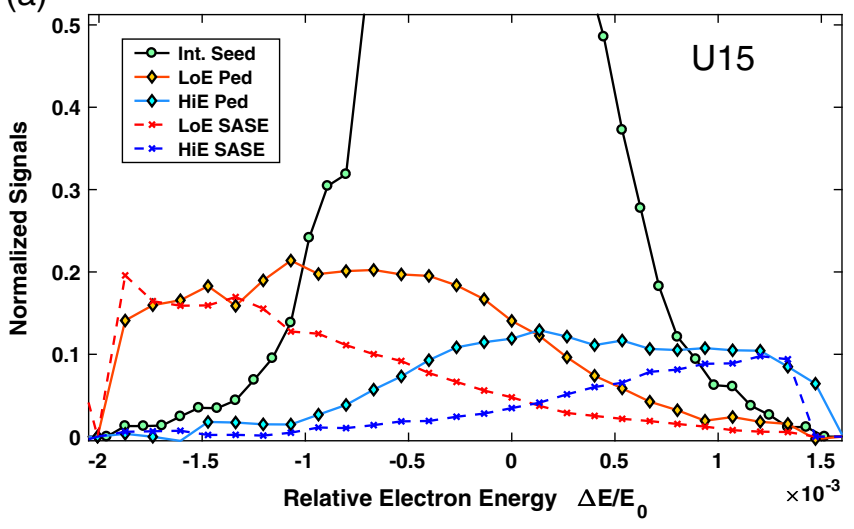

by the curves labeled "LoE SASE" and "HiE SASE." By contrast, when the normalized energy detuning remains relatively small compared to $\rho$, both the low energy and high energy spectral region pedestal are significantly stronger $(\sim O(0.05)$ or greater relative to the maximum seed strength) than the upper/lower spectral region SASE components.

This change in the ratio of pedestal region SASE to total pedestal strength strongly suggests that there is a significant component to the pedestal that is independent of the SASE background when the electron beam energy is close to that necessary for FEL resonance at $\lambda_{0}$. Furthermore, the fact that the strength of this non-SASE pedestal component relative to that of the amplified seed maximizes for shots with negligible $E_{B}$ detuning indicates that it must be driven at least in part by the amplified seed, i.e., a sideband-like phenomenon.

\section{PEDESTAL SPECTRAL CORRELATION PROPERTIES}

Inspection of individual spectra shows that both the integrated pedestal and details of its distribution can vary strongly from shot to shot. These variations arise from both the shot-to-shot electron beam energy jitter (e.g., see Fig. 12) and, as becomes apparent when considering only a narrow $E_{B}$ window, the stochastic behavior of the pedestal's individual components such as SASE (e.g., see Fig. 7). To further understand the pedestal's nature, we calculated, as a measure of the degree of linear correlation between the intensities of two spectral frequency components $\omega_{j}$ and $\omega_{k}$, the population Pearson correlation coefficient $r_{j k}$ :

$$
r_{j k}=\frac{s_{j k}^{2}}{s_{j j} s_{k k}}
$$

for various datasets, where

(b)

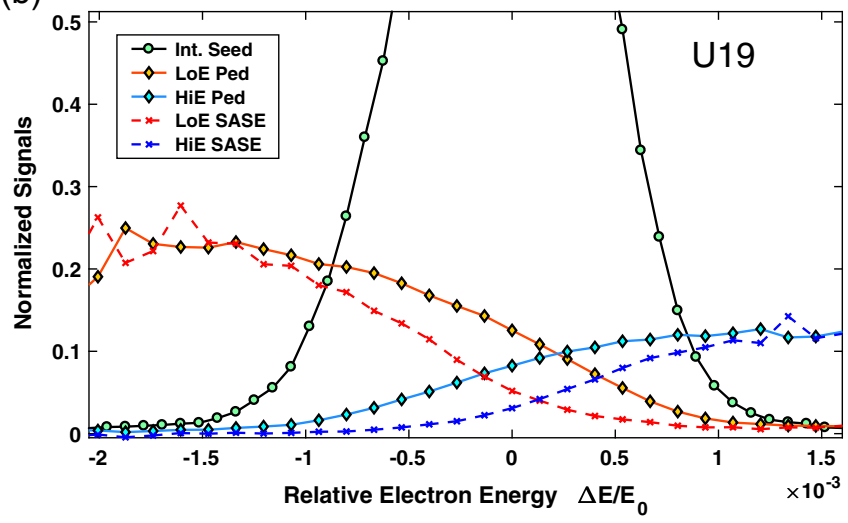

FIG. 12. Amplified seed strength ("Int. Seed"), upper ("HiE Ped”) and lower ("LoE Ped") pedestal wavelength region strength, and separate upper/lower SASE (i.e., blocked seed) strength as a function of electron beam detuning $\Delta E_{B}$ for two different effective undulator lengths in the November 2017 shift; the LH strength was $12 \mu \mathrm{J}$ in both cases. All curves are normalized to the peak value of the integrated seed with respect to $\Delta E_{B}$. 

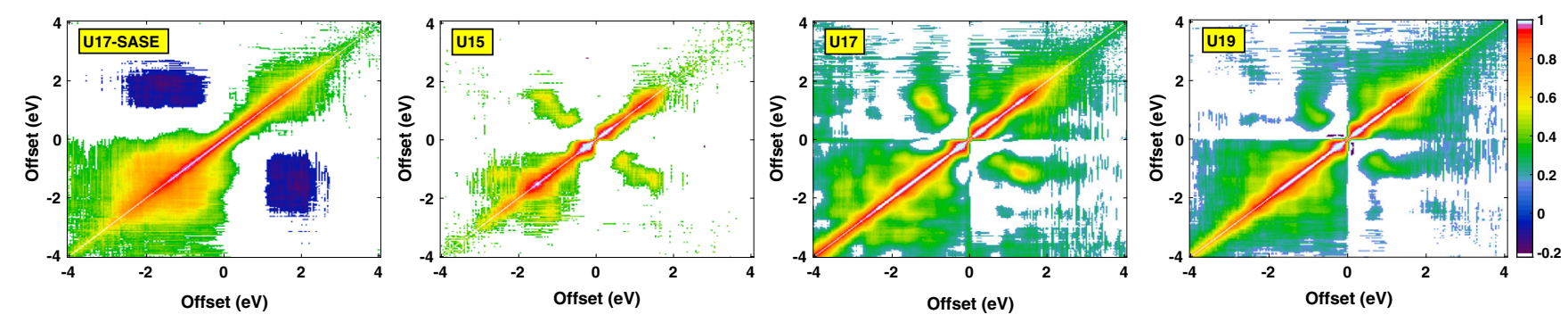

FIG. 13. False color representation of the Pearson correlation coefficients for intensity spectra from the November 2017 shift. The LH strength was $12 \mu \mathrm{J}$ in all cases and the individual shots were restricted to those both whose normalized electron beam energies were within $\pm 8 \times 10^{-5}$ of the mean (twice as large for the blocked seed U17 and self-seeded U15 cases to produce better statistics) and whose seed intensity $I_{i}$ satisfied $0.8 \leq I_{i} /\langle I\rangle \leq 1.25$. The latter filtering was done to minimize any PSF-induced correlations. To improve the visual contrast, only those correlation coefficients whose statistical significance exceeds $3.3 \sigma$ are shown in a nonwhite color. Negative correlation coefficients are clipped to a -0.2 minimum.

$$
s_{j k}^{2} \equiv \frac{1}{N-1} \sum_{i=1}^{N}\left[I_{i}\left(\omega_{j}\right)-\bar{I}\left(\omega_{j}\right)\right]\left[I_{i}\left(\omega_{k}\right)-\bar{I}\left(\omega_{k}\right)\right]
$$

is the sample covariance, the averages are defined in the usual sense as

$$
\bar{I}\left(\omega_{j}\right) \equiv \frac{1}{N} \sum_{i=1}^{N} I_{i}\left(\omega_{j}\right)
$$

and $N$ refers to the number of analyzed spectra in the sample. The values of $r_{j k}$ range from 0 , when the two spectral intensities exhibit no correlation, to \pm 1 when they are completely correlated or anticorrelated. Not surprisingly, this function has quite different properties for the SASE datasets as compared to those with self-seeding.

When considering the presaturation regime for pure SASE radiation, there have been a number of theoretical and experimental papers that address properties such as the cross-correlation function in both the time and frequency regime. In particular, the paper by Krinsky and Gluckstern [24] gives the expected probability distribution for $\left\langle I\left(\omega_{j}\right) I\left(\omega_{k}\right)\right\rangle /\left\langle I\left(\omega_{i}\right)\right\rangle\left\langle I\left(\omega_{k}\right)\right\rangle$ for an electron beam pulse whose coarse-grained envelope properties are both constant within the pulse and remain stationary in time. Inspection of their Eqs. (3.14), (3.17), (3.21), and (3.22) shows that for values of $\left|\omega_{j}-\omega_{k}\right| \leq \pi / \tau_{B}$ where $\tau_{B}$ is the pulse duration of a constant current electron beam, $I\left(\omega_{k}\right)$ has a significant positive correlation with $I\left(\omega_{j}\right)$, but for frequency separations $\left|\omega_{j}-\omega_{k}\right| \geq 10 / \tau_{B}$ the predicted correlation level drops to a percent or smaller. Numerically, this separation is approximately $200 \mathrm{meV}$ for the $\approx 100 \mathrm{fs}$ pulse duration corresponding to the values in Table I; given the effective smoothing by the PSF, the positive correlation will be extended another 200 or $300 \mathrm{meV}$ but should nonetheless be quite small for frequency separations of $750 \mathrm{meV}$ or greater. Importantly, the predicted correlation depends only upon the frequency separation and is independent of the nominal FEL resonant frequency $\omega_{R}$. These relationships hold true when $\tau_{B} \gg\left(2 \pi / \omega_{R}\right) \times\left(L_{G} / \lambda_{u}\right)$, the so-called FEL cooperation time.

Upon applying correlation analysis to the SASE data of Nov. 2017, the results follow the predictions of Ref. [24] so long as the data is filtered to include only shots whose $\left|\Delta E_{B}\right| \ll \rho$. If the filtering is relaxed such that the normalized energy acceptance encompasses a span $\geq O(0.2 \rho)$ or larger, a strong negative correlation coefficient appears when $\omega_{j}$ and $\omega_{k}$ lie on opposite sides of the frequency $\left\langle\omega_{R}\right\rangle$ corresponding to the mean $E_{B}$. This behavior is displayed in the far left plot of Fig. 13 (i.e., the purple splotches) and may be simply explained by the observation that those individual shots that have smaller beam energies will tend to have enhanced emission on the low frequency side of $\left\langle\omega_{R}\right\rangle$ and correspondingly reduced emission on the high frequency side, while the reverse is true for those shots with higher beam energies. In both cases this produces a negative cross-correlation coefficient that is symmetric with respect to $\left\langle\omega_{R}\right\rangle$.

When the correlation coefficients are calculated for the self-seeded datasets, such negative regions appear in general only when $\omega_{j}$ and $\omega_{k}$ lie on opposite sides of the monochromatized seed frequency $\omega_{0}$ and at least one or the other frequency is well within the bandwidth of the amplified seed $(\approx 300 \mathrm{meV})$. This presumably is a frequency pulling effect and is seen most strongly at effective undulator lengths where the seed is at or close to saturation, as is evident in the November 2017 U17 and U19 correlation maps shown in Fig. 13. A very different and positive $r_{j k}$ appears for radiation frequencies that lie symmetrically to either side of $\omega_{0}$, i.e., $\omega_{j}=\omega_{0}+\Delta \omega, \omega_{k}=\omega_{0}-\Delta \omega$. Each of the seeded datasets in Fig. 13 including U15 display such symmetric behavior, particularly at frequency offsets in the range to 0.5 to $1.5 \mathrm{eV}$.

This positive correlation between symmetric offset frequencies appears across multiple experimental shifts, as demonstrated in the lineouts plotted in Fig. 14. The magnitude of the positive correlation appears to be quite sensitive to the LH settings as it virtually disappears in 
(a)

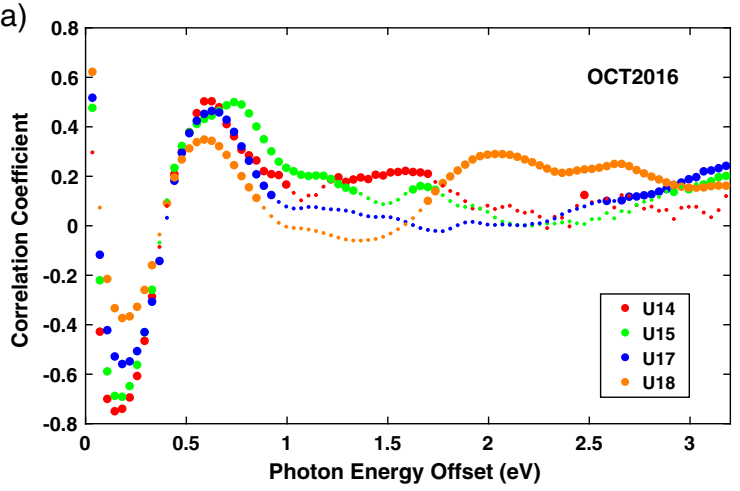

(b)

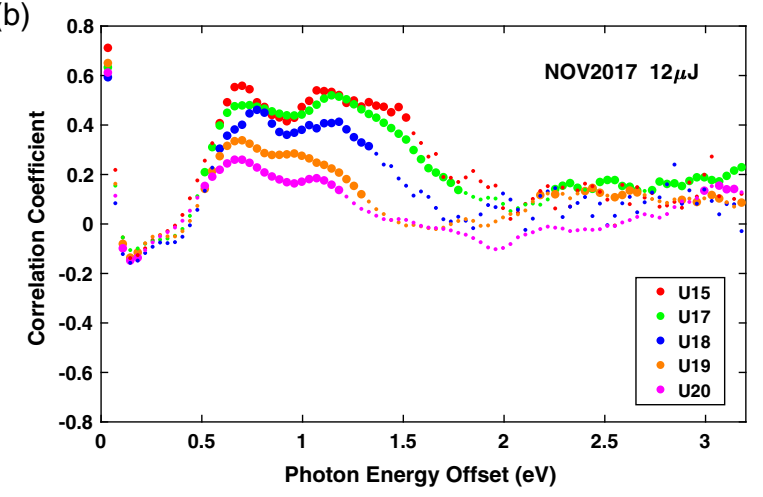

(c)

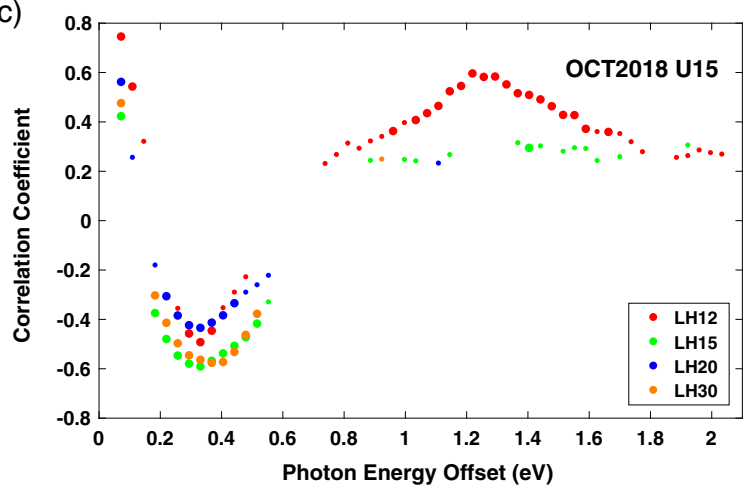

FIG. 14. Pearson correlation coefficients computed for the symmetric pair $\left[\omega_{0}-\Delta \omega, \omega_{0}+\Delta \omega\right]$. as a function of relative photon energy $\hbar \Delta \omega$ for (a) and (b) different effective undulator lengths for the 1-keV October 2016 and November 2017 shifts, respectively, and also (c) the 750-eV October 2018 shift at U15 for various LH energies. The larger dots correspond to data whose statistical significance $\mathrm{P}$-value is $\leq 10^{-3}$ or, equivalently, $\geq 3.3 \sigma$.

the Nov. 2017 shift for LH energies $\geq 20 \mu \mathrm{J}$, and the Oct. 2018 shift at U15 for energies $\geq 15 \mu \mathrm{J}$. Moreover, the positive correlation is not obvious beyond an effective undulator length of U17 in Nov. 2017 although the shot statistics are relatively poor compared to the earlier two 1-keV shifts.

A reasonable explanation for this symmetric frequencyoffset correlation pattern at low LH energies is that it arises from upper and lower sideband emission that was excited by long wavelength (i.e., $1 \mu \mathrm{m}$ ), longitudinal energy and/or density modulations on the electron beam similar to those modeled by Zhang et al. [15]. That paper's 1-D theoretical analysis predicted that the relative strength of such sideband emission (at least for frequency offsets $\Delta \omega \leq \rho \omega_{0}$ ) should grow with the effective undulator length squared. However, neither the experimental data shown in Fig. 8, where the wideband pedestal fraction grows only approximately linearly with $z$, nor that of Fig. 14, where the correlation coefficient generally decreases with increasing $z$, confirm such rapid growth. Perhaps some of the discrepancy can be explained by gain narrowing (which for wideband excitation would lead to a growth scaling closer to $z^{3 / 2}$ than $z^{2}$ dependence) and possibly various seed saturation effects that might begin as early as U17. Zhang et al. did predict that the lower sideband would be favored over that of upper sideband, an effect that would reduce the correlation coefficient (see their Fig. 2) for longer undulator lengths.

\section{CONCLUSIONS}

In this paper we have reported detailed experimental studies of soft X-ray self-seeding at the LCLS, focusing upon the behavior of the so-called pedestal emission. We find that the exponential growth rate of the amplified, monochromatized SASE signal is as expected, including detuning with respect to electron beam energy. The amplified seed has extremely good wavelength stability, considering both shotto-shot electron beam energy jitter at a given effective undulator length and the variation in $\langle\bar{\lambda}\rangle$ as a function of increasing undulator length, typically being only a few parts in $10^{5}$, as compared with electron beam energy jitter that is 20 times larger. As a corollary, frequency pulling effects on the amplified seed from variable electron beam energy appear to be very small.

At a given undulator length, both the strength and spectral details of the pedestal emission vary from shot to shot, indicating it does not originate in some pathological instrumental or diagnostic effect. We confirm that the pedestal-toseed fraction typically grows with effective undulator length and in some conditions (e.g., an ineffective laser heater) can exceed $15 \%$ as the FEL approaches saturation. The pedestal appears to have two distinct components. The first is "normal" SASE that we associate with wideband microbunching on the electron beam at very short wavelengths that fall near the resonant FEL wavelength (1.2 or $1.6 \mathrm{~nm}$ in this study) and which is present at shot noise levels as the electron beam reenters the undulator immediately following the SXR self-seeding chicane. For the experimental shifts (Nov. 2017 and Oct. 2018) with the best SASE diagnostics, the fractional strength of the SASE pedestal component is 0.07 or less for optimum LH energies.

The second pedestal component is sideband-like emission on either side of the amplified seed line. It appears to arise from much longer wavelength (i.e., $\lambda \sim 0.5-2 \mu \mathrm{m}$ ), longitudinal variations of the electron beam current and/or 
energy. Whereas the strength of the SASE pedestal component neither varies significantly with either the electron beam energy nor with the laser heater strength (over a restricted range of 12 to $30 \mu \mathrm{J}$ ), the sideband component essentially disappears when the magnitude of the normalized energy detuning approaches a value comparable to the FEL parameter $\rho$ or greater. Based on the results of two experimental shifts in which special attention was paid to proper alignment of the LCLS laser heater, this sideband pedestal component is also strongly damped by higher LH energies, with fractional strength values dropping from greater than 0.2 to less than 0.05 . Similar evidence for $\mathrm{LH}$ control of unwanted sideband emission around an amplified, harmonic-upshifted external seed has been found by the FERMI FEL team [25] for output wavelengths in the range $\sim 5-32 \mathrm{~nm}$. Finally, there is some evidence from multiple LCLS shifts for correlated, symmetric pedestal emission corresponding to $\hbar \Delta \omega \sim \pm 1-2 \mathrm{eV}$ on both sides of the amplified seed wavelength as would be expected from a long wavelength modulations of the electron beam energy. This symmetric emission has quite different spectral correlation properties than those of classic SASE.

In closing, we believe that the spectral purity of selfseeding when applied to high gain, soft x-ray FELs can be made much more effective both by optimizing control of electron beam longitudinal phase space via means such as a laser heater and also by maximizing the seed strength at entrance to post-monochromator undulator. For FELs such as the European XFEL or LCLS-II operated at very high repetition rates, this will likely pose some technological challenges with regards to monochromator optics survivability. Nonetheless, if the integrated pedestal emission can be limited to a reasonably small value (e.g., less than a 5\% fraction) at FEL saturation, the LCLS data considered here verify the basic promise of soft x-ray selfseeding, namely the production of high power output pulses with excellent wavelength stability and relatively narrow spectral bandwidths.

\section{ACKNOWLEDGMENTS}

We thank T. Maxwell and E. Allaria for useful discussions and the LCLS operators for their assistance and support during all the experimental shifts. This work was supported by U.S. Department of Energy Contract No. DE-AC02$76 \mathrm{SF} 00515$.

\section{APPENDIX A: SUMMARY OF EXPERIMENTAL PROCEDURES AND OPERATING CONDITIONS}

The LCLS SXR self-seeding data discussed above were taken over four separate experimental shifts beginning in 2015 and ending in 2018. There were many common features to these shifts but also some important differences which we now delineate in detail.

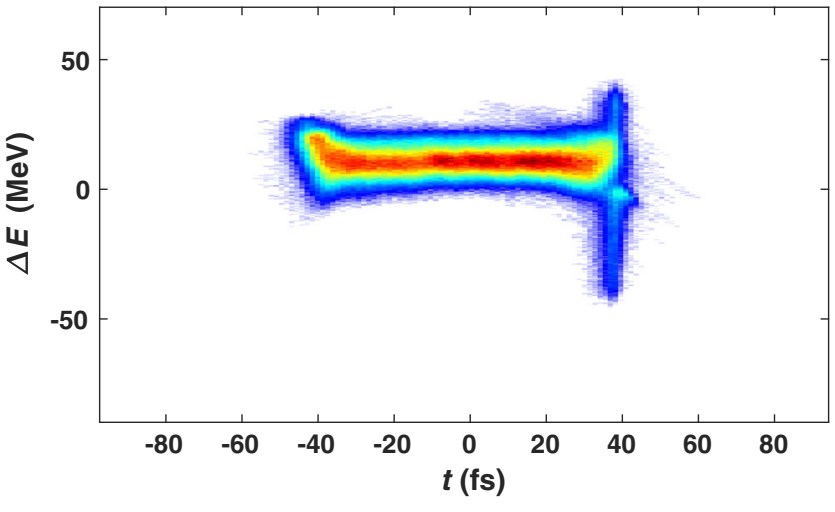

FIG. 15. Sample longitudinal phase space (FEL off) as measured at the CCD screen/camera system following the LCLS $\mathrm{X}$-band transverse deflector. The current was $\approx 1.3 \mathrm{kA}$ in the central \pm 35 fs portion of the electron beam.

For all shifts we attempted to optimize the electron beam longitudinal phase space for self-seeding, with a goal of a more or less temporally flat current and energy profile in the undulator that would produce high spectral brightness SASE from the first 8 undulator sections, but at integrated power levels that would be safely below the damage thresholds of the self-seeding monochromator optics positioned at the 9th undulator girder location (U9). This optimization involved first removing the head and tail current "horns" by reducing the nominal $250 \mathrm{pC}$ charge emitted from the cathode down to $140 \mathrm{pC}$ via collimation in the first LCLS bunch compressor BC1 [26]. We then attempted to minimize simultaneously both the observed SASE bandwidth on the post-undulator spectrometer and the electron beam phase space curvature as measured on the diagnostic screen that follows the X-band rf transverse deflector [27]. This minimization was done primarily by manipulating the L1 linac section phase upstream of BC1 while maintaining an overall post-BC2 compressed current of $\approx 1.3 \mathrm{kA}$, a value having been shown empirically to be a good operating point for self-seeding LCLS at SXR energies. A characteristic image of the longitudinal phase space is shown in Fig. 15.

Beside the grating-based monochromator, an electron beam chicane also occupies the U9 space. This chicane delays the electron beam to provide temporal overlap with the monochromatized SASE x-rays that seed the downstream radiator undulator segments beginning at location U10. The chromatic dispersion associated with the chicane's $\approx 0.5$ ps delay together with the incoherent energy spread on the electron beam should wash out any microbunching structures at XUV and shorter wavelengths created by upstream SASE and MBI processes. Consequently, we believe SASE that develops at location U10 and beyond should be initiated at a level close to that expected from nominal shot noise statistics.

We operated the monochromator without the use of an exit slit. Since the entire SASE spectrum is passed by the 
monochromator in this scenario, the effective seeding bandwidth is determined by the transverse overlap at the $\mathrm{U} 10$ reinsertion position between the dispersed radiation and the electron beam; i.e., the electron beam acts as its own exit slit [28]. Operationally, at $1.0 \mathrm{keV}$ FEL resonance this leads to an acceptance of $\approx 200 \mathrm{meV}$ (FWHM), significantly smaller than the $\sim 1-2 \mathrm{eV}$ SASE bandwidth at the end of U8. Presuming a longitudinal Gaussian shape, the Fourierlimited radiation pulse length for this acceptance is roughly $9 \mathrm{fs}$, a value much shorter than the $\approx 100$ fs duration of the electron beam pulse. We therefore expect that the postmonochromator undulator segments are seeded with multiple temporal and frequency radiation spikes. As mentioned before, the detailed shot-to-shot statistics of the seed and pedestal radiation are currently under study [22].

The x-ray spectra were measured downstream of the LCLS undulator hall using the single-shot spectrometer system developed for the SXR materials science instrument. This system consists of a varied-line-spacing (VLS) grating monochromator and a spherical focusing mirror [29]. The spectrometer detector includes a cerium-doped, yttrium-aluminum-garnet (Ce:YAG) crystal that scintillates in the visible when activated by x-rays, a 45 degree mirror, and an optical camera (optical lens and $1024 \times 1024$ pixel CCD camera combination). The 100 lines per millimeter VLS grating used for these shifts and an upstream spherical mirror together create a vertical focus with a magnification setting of 37 and $17 \mathrm{meV}$ per pixel at the output $\mathrm{CCD}$ for $1 \mathrm{keV}$ and $750 \mathrm{eV}$ photon energies, respectively. The empirically-determined point spread function (PSF) of the overall camera system had a FWHM of $\approx 270 \mathrm{meV}$ at $1.0 \mathrm{keV}$ (see Appendix $\mathrm{B}$ for discussion of PSF measurements). In order to enhance the signal-to-noise ratio of the final spectra, we numerically reduced the $2 \mathrm{D}$ CCD spectrum to a one dimensional signal $S(\lambda)$ by integrating over $\approx 400$ pixels in the non-dispersive direction. The last two shifts also took special CCD background datasets in which the electron beam was suppressed; backgrounds for the earlier June 2015 and October 2016 shifts were estimated on a shot-by-shot basis by presuming a simple linear relation between the far red and blue wavelength regions where the seed, SASE, and pedestal signals should be very small. The spectral distribution function of a typical self-seeded data set, following binning of individual shots according to their electron beam energy $E_{B}$ as determined from measured X-plane deflection at LCLS location LTU250, is displayed in the left plot of Fig. 2; regions corresponding to the amplified seed, "normal" SASE, and pedestal are readily apparent.

The four experimental shifts differed in some important details. First, the November 2017 and October 2018 shifts were the only ones where the LH was carefully aligned, both spatially and temporally, to ensure optimal MBI suppression. In addition, these were also the only shifts where the LH energy and thus its contribution to the upstream value of the electron beam's incoherent $\sigma_{E}$ was systematically changed to examine the self-seeding pedestal's sensitivity to possible MBI-induced longitudinal phase space structures. To do so required special operational permission to bypass machine protection controls that safeguard against potential damage to the self-seeding monochromator optics. There was no undulator magnetic strength $K$ tapering beyond that needed for spontaneous emission and resistive-wall wakefield losses. Via appropriate use of the gas absorption attenuation cell, we minimized saturation effects at the post-undulator diagnostic spectrometer CCD. Importantly, these last two shifts also obtained additional datasets in which the seed was blocked using the monochromator exit slit holder before its re-entry to U10. In principle such blocked seed datasets (e.g., see panel (b) of Fig. 2) measure the properties of "pure" SASE growth downstream of the seeding monochromator. Typically we took 3000-6000 seeded shots and 1000 SASE shots for each LH energy. During the October 2018 shift at $750 \mathrm{eV}$, studies of SASE and pedestal sensitivity to LH energy were conducted only at an effective U15 undulator length. As discussed in Sec. III C, the numerical ratio of the wavelength-integrated SASE to amplified seed emission for these two shifts ranges from $\sim 0.10$ to 0.20 depending upon undulator location and LH energy.

The earlier June 2015 and October $20161.0 \mathrm{keV}$ shifts appear to have been self-seeded at a power level at U10 2-3× greater than the last two shifts and reached FEL saturation by $\approx \mathrm{U} 18$. Consequently they proved to be particularly useful in determining amplified seed properties as spectral profiles could be determined as early as the end of segment U11. These shifts employed undulator strength tapering beyond U17 to increase the amplified seed strength. The October 2016 shift was notable in terms of the very large number $(\approx 15000)$ of seeded spectra acquired for each effective undulator length. This allowed quite narrow electron beam range filtering to examine properties such as frequency pulling and spectral correlation properties. Unfortunately, the post-undulator gas absorption cell was not used in these early shifts, resulting in spectrometer CCD saturation effects by location U17 and beyond at the highest amplified seed intensities. Furthermore, we suspect ex post facto that the $\mathrm{LH}$ focal spot was not well aligned transversely with the electron beam orbit. No blocked seed datasets were taken in these shifts, so there are no highly reliable quantitative measures of the SASE contribution to pedestal emission.

\section{APPENDIX B: DETERMINATION OF THE EFFECTIVE POINT SPREAD FUNCTION}

Without detailed knowledge of the effective point spread function (PSF) $P\left(\lambda, \lambda^{\prime}\right)$ of the downstream spectrometer/ camera system, there is quantitative uncertainty regarding the pedestal strength on a given shot. During the extended 


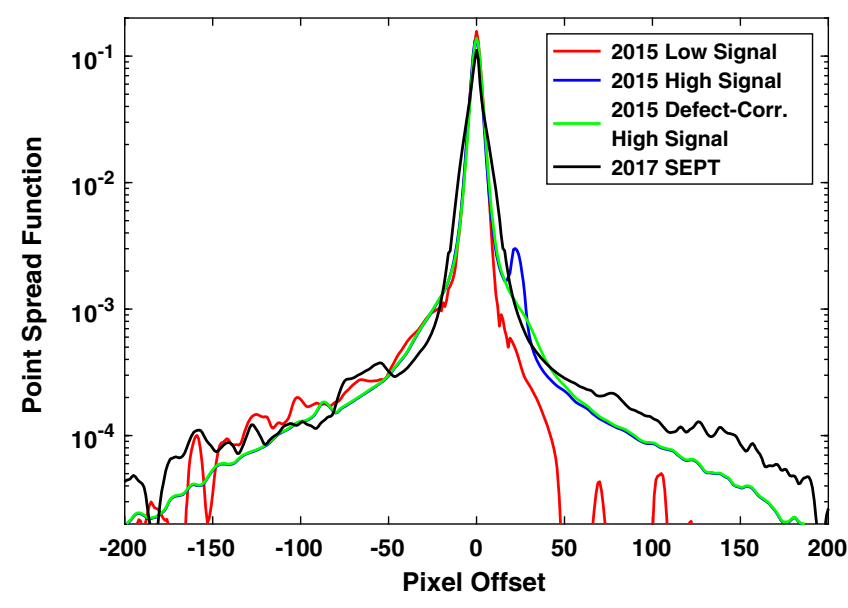

FIG. 16. Zero-order "direct" PSF functions obtained from 3 different datasets obtained in 2015 (low and high FEL radiation signals) and September 2017. The green line labeled "DefectCorr." includes a subjective correction of a CCD damage defect affecting the high radiation signal 2015 PSF data at a pixel offset of +30 .

course of these experimental studies, we attempted to measure the PSF by two different means.

The first was essentially direct, operating the spectrometer in zero order and focusing the output FEL radiation beam as tightly as possible at the grating location. In principle this produces a $\delta$-function-like source at a single source wavelength $\lambda_{*}$ and the output CCD signal $S(\lambda)$ should scale linearly with $P\left(\lambda, \lambda_{*}\right)$. A previous zero order measurement was made during the SXR spectrometer commissioning as shown in Fig. 6 of Ref. [29]; one sees that even on a linear scale the profile contains non-Gaussian tails stretching many FWHM distances away from the central region. Because there is likely some measurement sensitivity both to the actual focal spot size at the spectrometer slit and to the transfer optics between the YAG screen and CCD recording system, it would not be surprising if the PSF can vary over time periods of months to years.

For the current study we analyzed two more recent sets of such zero order measurements, one obtained in 2015 that included both low and high radiation intensity data, and a later one made in September 2017. The results are plotted in Fig. 16; due to sensitivity to the details of the procedure that removes the CCD background signal, derived values for $P\left(\lambda, \lambda_{*}\right)$ below $5 \times 10^{-5}$ should be treated with caution, especially for the 2015 low signal data. Table II gives various FWHM measures, i.e., the "classic" $50 \%$ of peak width, the $50 \%$ width for a numerically optimized 1D Gaussian fit, and finally the "effective" FWHM that contains $76.1 \%$ of the integrated signal, as would be true for a perfect Gaussian profile. With the exception of the effective FWHM value, which is significantly larger for the 2017 PSF measurement, the values are relatively close to one another suggesting that the core portion of PSF is reasonably well-modeled by
TABLE II. Zero-order PSF widths (pixels).

\begin{tabular}{lccc}
\hline \hline Dataset & FWHM & $\begin{array}{c}\text { FWHM } \\
\text { (Gauss. Fit) }\end{array}$ & $\begin{array}{c}\text { FWHM } \\
\text { (Effective) }\end{array}$ \\
\hline 2015 Small Signal & 5.0 & 5.8 & 8.0 \\
2015 Large Signal & 5.8 & 6.0 & 8.9 \\
2017 Sept. & 6.1 & 8.6 & 14.5 \\
\hline \hline
\end{tabular}

a Gaussian profile. For comparison with the $1-\mathrm{keV}$ shift pedestal strengths measured in the spectral regions $\pm[0.744 .48]$ eV (i.e., Fig. 8), the average of the corresponding pixel-integrated large signal 2015 and Sept. 2017 PSF data is 0.056 with a nominal error of \pm 0.002 .

Our second method to determine the effective PSF was much more indirect and empirical in nature. Here we exploited the fact that the overwhelming amount of PSF-caused leakage into the low intensity, pedestal wavelength regions originates from the relatively narrow bandwidth, much stronger intensity, amplified seed signal. Consequently, a good estimate of the PSF should be obtainable by looking at the shot-to-shot correlation between signals in the pedestal region and the integrated seed region strength. To minimize PSF overestimation errors due to CCD saturation effects or contamination by true pedestal signals (whose sideband-like component is expected to be positively correlated with the amplified seed signal), we used the following set of strategies: First, we considered only shots in datasets corresponding to effective undulator lengths for which the peak amplified seed signal was at least $100 \times$ greater than the apparent noise level and for which there also appeared to be no or very small saturation effects. Second, to determine correlations for wavelengths $\lambda$ greater(smaller) than $\lambda_{o}$, we considered only those individual shots whose normalized electron energy $\Delta E_{B} /\left\langle E_{B}\right\rangle$ offset from FEL resonance was positive(negative) and whose absolute value $\geq \rho$. This biased energy filtering strongly reduces both SASE contamination at $\lambda$ (i.e., consider the SASE contribution in Figs. 11 and 12) and the relative magnitude of any sideband-like pedestal contribution because both will have much smaller exponential gain than that of the seed.

Exploiting all these strategies and assumptions, we then did a linear regression analysis to extract a PSF-like signal $P\left(\lambda, \bar{\lambda}_{o}\right)$ where we adopt the symbol $\bar{\lambda}_{o}$ to signify an effective averaging over the central seed region. To compare this empirically determined PSF with the direct, zero-order measurements, we convolved the latter with a 2.54 pixel-wide (RMS) Gaussian profile seed (equivalent to a FWHM of $221 \mathrm{meV}$ for the applicable grating setting at $1.0 \mathrm{keV}$ ). This particular value, following convolution with an average of the large signal 2015 and 2017 zeroorder PSF's, was the best match to the amplified seed's apparent FWHM as shown in Fig. 5. Figure 17 shows the resulting curves; one sees reasonable but obviously not exact agreement between the empirical and the convolved 


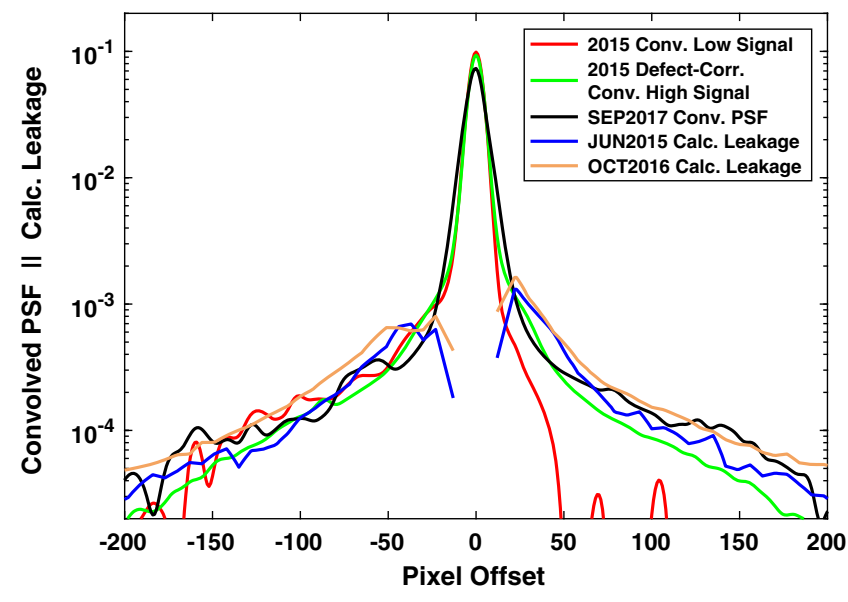

FIG. 17. Zero-order "direct" PSF functions after convolution with a 2.79 pixel (RMS) Gaussian plotted together with the indirectly determined PSF "leakage" functions in the pedestal wavelength region for the June 2015 and October 2016 experimental shifts.

zero-order determinations. The leakage fractions calculated for the June 2015 and Oct. 2016 shifts into the pedestal spectral regions used in Sec. III are 0.064 and 0.083 , respectively. These values are approximately 0.015 larger than the Gaussian-convolved, zero-order PSF signals discussed above, suggesting a possibly incomplete removal of all pedestal signal during the empirical PSF determination.

Despite the reasonable agreement between the direct and indirect PSF estimates, in the end we decided to be highly cautious and not apply any PSF corrections to the data shown in the main body of this paper with the exception of the enclosed spectral energy fraction plotted in Fig. 5. This caution arose from multiple concerns: the time separation between the date of the zero-order measurements and the 2016-2018 experimental data shifts during which the true PSF might change, the inexactness of the empirical determination method and its sensitivity to true pedestal contamination and, most importantly, the fact that the apparent, non-PSF-corrected, wavelength integrated pedestal fraction beyond $0.74 \mathrm{eV}$ often exceeds 0.2 , especially for datasets where the LH is not highly effective, a value dominating the apparent PSF leakage effects.

Nonetheless, the magnitude of the directly measured PSF, even when considering a spectral region separated by greater than $3 \sigma_{\lambda}$ from the central seed wavelength $\lambda_{o}$, suggests that one must apply appropriate caution when attempting to measure quantitatively a true pedestal whose integrated strength is $10 \%$ or less of an amplified seed. Accurate measurements become even more difficult for wavelength regions even closer than $3 \sigma_{\lambda}$ because errors in PSF-deconvolution can lead to serious overor underestimates of a pedestal, especially one that has a component that positively correlates with seed strength.

\section{APPENDIX C: FREQUENCY PULLING EFFECTS}

As mentioned in Sec. II C, the mean wavelength $\bar{\lambda}$ of the central line position at a given effective undulator length is more or less constant when considering a restricted subset of individual shots whose e-beam energy $E_{B}$ lies within a narrow region centered on the FEL resonance. However, when considering all shots obtained irrespective of $E_{B}$, some of the experimental shifts showed a small but definite shot-to-shot correlation between $\bar{\lambda}$ and $E_{B}$, i.e., a "frequency pulling" effect. For a warm temperature linac such as LCLS that has non-negligible energy jitter, serious frequency pulling could limit the average FEL spectral brightness.

A relatively strong example of this is shown in Fig. 18(a) that plots the distribution function $\left(\Delta E_{B} / E_{o}, \Delta \bar{\lambda} / \lambda_{o}\right)$ for the U15 datasets from the October 2016 shift. Here a change
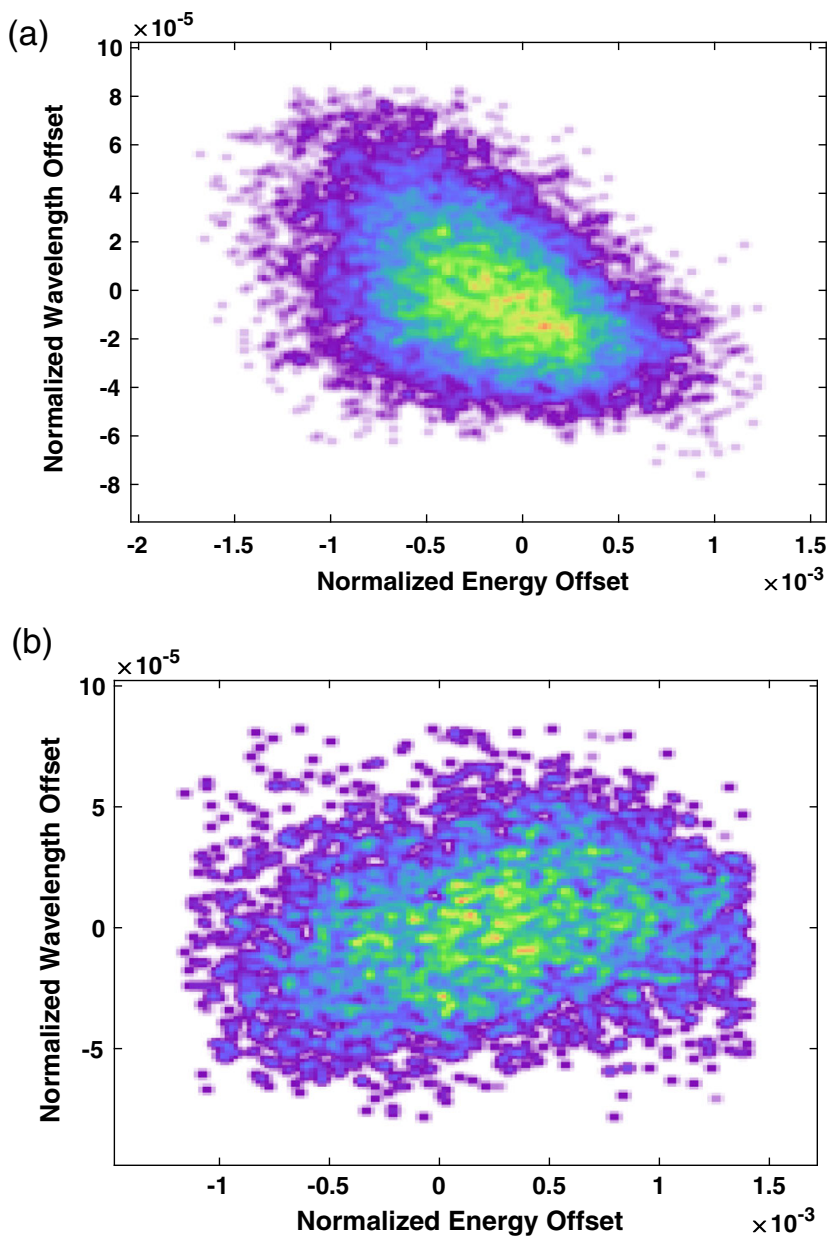

FIG. 18. False color distribution functions for the relative mean wavelength at effective undulator location U15 for the central amplified seed line as a function of relative electron beam energy, as determined from (a) 11463 individual shots of the October 2016 shift (b) 8680 shots of the June 2015 shift. 
of $\approx+2 \times 10^{-3}$ in $\Delta E_{B} / E_{o}$, results in a shift of $\approx-9 \times$ $10^{-5}$ in $\Delta \bar{\lambda} / \lambda_{o}$. The equivalent slope is $\approx 45$ times less in magnitude than would be true for SASE radiation, again displaying how self-seeding operation strongly reduces shot-to-shot wavelength jitter. However, equivalent data taken in June 2015 also at U15 shown in Fig. 18(b) does not show such an obvious correlation.

Interestingly, the correlation slope in the October 2016 data appears to increase as a function of effective undulator length, especially for shots with more positive $\Delta E_{B}$. There are several possible explanations for this increase: (1) An increase of SASE strength relative to the amplified seed at wavelengths $\lambda<\lambda_{0}$ for individual shots whose $E_{B}(z)>$ $\left\langle E_{B}(z)\right\rangle$; this effect becomes stronger as the amplified seed begins to saturate. The taper in undulator strength $K$ used in this shift might also have pulled the centroid of any underlying SASE emission bluewards. (2) The shift of the peak of the gain curve in $\lambda$ for off-energy shots leads to preferential amplification of seed spectral power to one side of $\lambda_{0}$. (3) Possible linear and higher order chirps on the electron beam energy together with slippage between the radiation and electron pulse could also pull the temporal average of amplified seed emission toward one or the other side of $\lambda_{0}$.

We attribute the observed changes from one experimental shift to another in the correlation properties between $\bar{\lambda}$ and $E_{B}$ to variations in the details of the longitudinal phase space distribution of the electron beam, in particular the magnitude of linear and higher order energy chirps. There might also be some sensitivity to the taper profile, but this was not explored in any detail. We note that similar observations of self-seeding wavelength stability and the lack of significant frequency pulling can be inferred from the LCLS hard x-ray results previously reported by Welch et al. [30]. Finally, the presence and sensitivity of such frequency pulling could in principle be useful as a linac tuning diagnostic while optimizing the average spectral brightness.

[1] W. Ackermann et al., Operation of a free-electron laser from the extreme ultraviolet to the water window, Nat. Photonics 1, 336 (2007).

[2] P. Emma et al., First lasing and operation of an Angstromwavelength free-electron laser, Nat. Photonics 4, 641 (2010).

[3] T. Ishikawa et al., A compact x-ray free-electron laser emitting in the sub-Angstrom region, Nat. Photonics 6, 540 (2012).

[4] H.-S. Kang et al., Hard x-ray free-electron laser with femtosecond-scale timing jitter, Nat. Photonics 11, 708 (2017).

[5] W. Decking and H. Weise et al., Commissioning of the European XFEL Accelerator, in Proceedings of the 8th International Particle Accelerator Conference Proceedings (IPAC2017), Copenhagen, Denmark (ESS \& MAX-IV,
Sweden and Aarhus Univ., Denmark, 2017), p. 1, https:// accelconf.web.cern.ch/AccelConf/ipac2017/papers/moxaa1 .pdf.

[6] J. Feldhaus, E. Saldin, J. Schneider, E. Schneidmiller, and M. Yurkov, Possible application of x-ray optical elements for reducing the spectral bandwidth of an x-ray SASE FEL, Opt. Commun. 140, 341 (1997).

[7] G. Geloni, V. Kocharyan, and E. Saldin, A novel selfseeding scheme for hard x-ray FELs, J. Mod. Opt. 58, 1391 (2011).

[8] J. Amann et al., Demonstration of self-seeding in a hardX-ray free-electron laser, Nat. Photonics 6, 693 (2012).

[9] D. Ratner et al., Experimental Demonstration of a Soft X-Ray Self-Seeded Free-Electron Laser, Phys. Rev. Lett. 114, 054801 (2015).

[10] W. Fawley and G. Penn, Thoughts on spectral broadening effects in FERMI and other FELs based upon seeded harmonic generation, Sincrotrone Trieste Tech. Report No. ST/F-TN-06/07, 2006; see also W. M. Fawley, G. Penn, and A. Zholents, Output Bandwidth Effects in Seeded, Harmonic Cascade FELs, in Proceedings of the 28th International Free Electron Laser Conference (FEL06), Berlin Germany (BESSY), 2006, p. 500; talk slides available at http://epaper.kek.jp/f06/TALKS/ THAAU01_TALK.PDF.

[11] A. Lutman, G. Penco, P. Craievich, and J. Wu, Impact of an initial energy chirp and an initial energy curvature on a seeded free electron laser: free electron laser properties, J. Phys. A 42, 085405 (2009).

[12] B. Jia, Y. K. Wu, J. J. Bisognano, A. W. Chao, and J. Wu, Influence of an imperfect energy profile on a seeded free electron laser performance, Phys. Rev. Accel. Beams 13, 060701 (2010).

[13] A. Marinelli, C. Pellegrini, L. Giannessi, and S. Reiche, Comparative study of nonideal beam effects in high gain harmonic generation and self-seeded free electron lasers, Phys. Rev. Accel. Beams 13, 070701 (2010).

[14] R. Lindberg, Note on FEL seeding in the presence of electron beam non-uniformities, unpublished work in 2012; issued as ANL Technical Report No. AOP-TN2015-028, 2015

[15] Z. Zhang, R. Lindberg, W. M. Fawley, Z. Huang, J. Krzywinski, A. Lutman, G. Marcus, and A. Marinelli, Microbunching-instability-induced sidebands in a seeded free-electron laser, Phys. Rev. Accel. Beams 19, 050701 (2016).

[16] K. Zhang, L. Zeng, Z. Qi, C. Feng, and D. Wang, Eliminating the microbunching-instability-induced sideband in a soft x-ray self-seeding free-electron laser, Nucl. Instrum. Methods Phys. Res., Sect. A 882, 22 (2018).

[17] E. L. Saldin, E. A. Schneidmiller, and M. V. Yurkov, Klystron instability of a relativistic electron beam in a bunch compressor, Nucl. Instrum. Methods Phys. Res., Sect. A 490, 1 (2002).

[18] Z. Huang, M. Borland, P. Emma, J.Wu, C. Limborg, G. Stupakov, and J. Welch, Suppression of microbunching instability in the linac coherent light source, Phys. Rev. Accel. Beams 7, 074401 (2004).

[19] D. Ratner, C. Behrens, Y. Ding, Z. Huang, A. Marinelli, T. Maxwell, and F. Zhou, Time-resolved imaging of the 
microbunching instability and energy spread at the Linac Coherent Light Source, Phys. Rev. Accel. Beams 18, 030704 (2015).

[20] J. Qiang, Y. Ding, P. Emma, Z. Huang, D. Ratner, T. O. Raubenheimer, M. Venturini, F. Zhou, Start-to-end simulation of the shot-noise driven microbunching instability experiment at the Linac Coherent Light Source, Phys. Rev. Accel. Beams 20, 054402 (2017).

[21] E. Roussel, E. Ferrari, E. Allaria, G. Penco, S. Di Mitri, M. Veronese, M. Danailov, D. Gauthier, and L. Giannessi, Multicolor High-Gain Free-Electron Laser Driven by Seeded Microbunching Instability, Phys. Rev. Lett. 115, 214801 (2015).

[22] Z. Zhang and G. Marcus (to be published).

[23] W. M. Fawley, A User Manual for GINGER, LBNL Report No. LBNL-49625, 2002; see also W. M. Fawley, An Enhanced GINGER Simulation Code with Harmonic Emission and HDF5 IO Capabilities, in Proceedings of the 28th International Free Electron Laser Conference (FEL06), Berlin Germany (BESSY), 2006), p. 218, https://accelconf .web.cern.ch/AccelConf/f06/papers/mopph073.pdf.

[24] S. Krinsky and R. L. Gluckstern, Analysis of statistical correlations and intensity spiking in the self-amplified spontaneous-emission free-electron laser, Phys. Rev. Accel. Beams 6, 050701 (2003).

[25] E. Allaria, L. Gianessi, and S. Spampinati (private communication); see also S. Spampinati et al., Commissioning of the FERMI@ELETTRA Laser Heater, in Proceedings of the 34th International Free Electron Laser Conference (FEL12), Nara, Japan (SPring-8 and Kyoto Univ., Japan, 2012), p. 177, https://accelconf.web.cern.ch/AccelConf/ FEL2012/papers/mopd58.pdf.

[26] Y. Ding et al., Beam shaping to improve the free-electron laser performance at the Linac Coherent Light Source, Phys. Rev. Accel. Beams 19, 100703 (2016).

[27] C. Behrens et al., Few-femtosecond time-resolved measurements of x-ray free-electron lasers, Nat. Commun. 5, 3762 (2014).

[28] S. Serkez, J. Krzywinski, Y. Ding, and Z. Huang, Soft x-ray self-seeding simulation methods and their application for the Linac Coherent Light Source, Phys. Rev. Accel. Beams 18, 030708 (2015).

[29] P. Heimann et al., Linac Coherent Light Source soft x-ray materials science instrument optical design and monochromator commissioning, Rev. Sci. Instrum. 82, 093104 (2011).

[30] J. Welch, F.-J. Decker, J. Hastings, Z. Huang, A. Lutman, M. Messerschmidt, and J. L. Turner, Comparison of hard X-ray self-seeding with SASE after a monochromator at LCLS, in Proceedings of the 34th International Free Electron Laser Conference (FEL 12), Nara, Japan (SPring-8 and Kyoto Univ., Japan, 2012), p. 217, https:/accelconf.web.cern.ch/AccelConf/FEL2012/papers/ tuob04.pdf. 